\title{
Modified Chiral Triazolium Salts for Enantioselective Benzoin Cyclization of Enolizable Keto-aldehydes: Synthesis of (+)- Sappanone B
}

\author{
Hiroshi Takikawa and Keisuke Suzuki* \\ [*] Department of Chemistry, Tokyo Institute of Technology, SORST-JST Agency, \\ 2-12-1 O-okayama, Meguro-ku, Tokyo 152-8551 (Japan)
}

Supporting Information

Table of contents S1

General methods $\mathrm{S} 2$

Procedure and spectral data S3

${ }^{1} \mathrm{H}$ and ${ }^{13} \mathrm{C}$ spectra S17 


\section{General Methods}

All reactions utilizing air- or moisture-sensitive reagents were performed in dried glassware under an atmosphere of dry argon and nitrogen. Ethereal solvents (anhydrous; Kanto Chemical Co., Inc.) were used as received. Toluene was distilled from $\mathrm{CaH}_{2}$ prior to use. $\mathrm{CH}_{2} \mathrm{Cl}_{2}$ was distilled successively from $\mathrm{P}_{2} \mathrm{O}_{5}$ and $\mathrm{CaH}_{2}$, and stored over $4 \mathrm{~A}$ molecular sieves. DBU was distilled from $\mathrm{BaO}$. $\mathrm{Et}_{3} \mathrm{~N}$ was distilled from $\mathrm{CaH}_{2}$ and stored over $\mathrm{KOH}$. Other reagents were used without further purification. Thin layer chromatography (TLC) was performed on Merck precoated plates (silica gel $60 \mathrm{~F} 254$, Art 5715, $0.25 \mathrm{~mm}$ ) and were visualized by fluorescence quenching under UV light or by staining with phosphomolybdic acid. Silica-gel preparative thin-layer chromatography (PTLC) was performed using plates prepared from Merck Kieselgel 60 PF 254 (Art 7747). Silica-gel column chromatography was performed on silica gel $60 \mathrm{~N}$ (Spherical, neutral, 23-210 $\mu \mathrm{m}$ ) from Kanto chemical. Melting point (m.p.) determinations were performed using a Yanaco MP-500 instrument and are uncorrected. ${ }^{1} \mathrm{H}$ NMR and ${ }^{13} \mathrm{C}$ NMR were measured on a JEOL JMN Lambda-300 or Lambda-400 spectrometer. Chemical shifts are expressed in parts per million (PPM) downfield from internal standard (tetramethylsilane, $0.00 \mathrm{ppm}$ ), and coupling constants are reported as hertz $(\mathrm{Hz})$. Splitting patterns are indicated as follows: br, broad; s, singlet; d, doublet; t, triplet; q, quartet; m, multiplet. Infrared (IR) spectra were recorded on a Jasco IRA-202 spectrophotometer or Perkin Elmer Spectrum 100 spectrometer. Attenuated Total Reflectance Fourier Transform Infrared (ATRFTIR) spectra were recorded on a Perkin Elmer 1600 FTIR. Optical rotations $\left([\alpha]_{D}\right)$ were measured on a JASCO DIP-1000 polarimeter. High performance liquid chromatography (HPLC) analyses were performed using a JASCO CO-2060 plus for column thermostat, UV-2075 plus for UV/VIS detector, CD-2095 plus for chiral detector, PU-2089 plus for HPLC pump. Enantiomeric excesses were assessed by HPLC analysis on a chiral stationary phase, CHIRALPAK $^{\circledR}$ AD-H or CHIRALCEL ${ }^{\circledR}$ OD-H or CHIRALPAK ${ }^{\circledR}$ IA (Daicel Chemical Ind., Ltd., $\phi 0.48 \mathrm{~mm} \times 25 \mathrm{~cm}$ ). High resolution mass spectra (HRMS) were obtained with a JEOL JMS700 spectrometer.

\section{Preparation of oxalated silica gel}

A mixture of silica gel $(200 \mathrm{~g})$ and water solution of oxalic acid $(0.25 \mathrm{M}, 500 \mathrm{~mL})$ was stirred with mechanical stirrer for $9 \mathrm{~h}$. The resulting slurry was poured into a column equipped with a glass filter. Ion-exchange water was passed through the column until the waste turned into neutral. The resulting gel was dried in an oven at $120^{\circ} \mathrm{C}$ for $3 \mathrm{~h}$ to give oxalated silica gel. ${ }^{[1]}$

[1] For oxalated silica gel, see; Cameron, D. W.; Riches, A. G. Aust. J. Chem. 1997, 50, 409-424. 


\section{Preparation of chiral triazolium salts $3 \mathrm{c}-\mathbf{3 f}$}

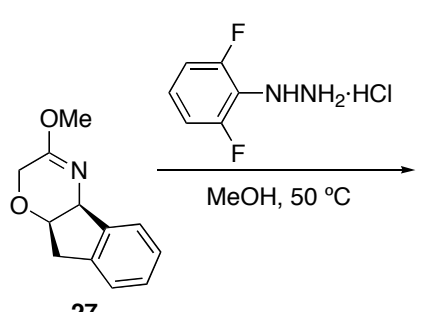

27

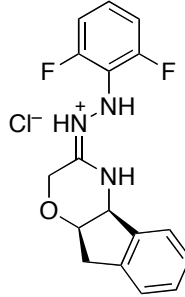

28

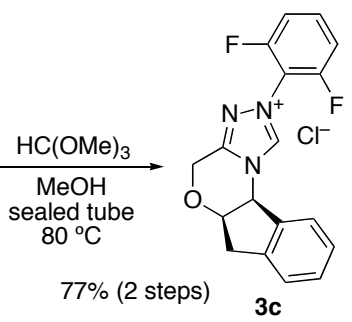

To a solution of iminoether $\mathbf{2 7}^{[2]}(173 \mathrm{mg}, 0.851 \mathrm{mmol})$ in $\mathrm{MeOH}(4.5 \mathrm{~mL}), 2,6$ difluorophenylhydrazine hydrochloride $(154 \mathrm{mg}, 0.851 \mathrm{mmol})$ was added at room temperature. The mixture was warmed to $50{ }^{\circ} \mathrm{C}$ and stirred for $3 \mathrm{~h}$. After cooling to room temperature, the solvents were evaporated under reduced pressure. The residue was washed with $\mathrm{Et}_{2} \mathrm{O}$ and dried in vacuo to afford hydrazone hydrochloride 28 . In a flame-dried $20 \mathrm{~mL}$ sealed tube was placed hydazone hydrochloride 28 in trimethyl orthoformate $(3.0 \mathrm{~mL})$ and methanol $(1.5 \mathrm{~mL})$. The reaction mixture was stirred for $27 \mathrm{~h}$ at $80{ }^{\circ} \mathrm{C}$. After the solvents were removed in vacuo, precipitation from $\mathrm{CH}_{3} \mathrm{CN} / \mathrm{Et}_{2} \mathrm{O}$ gave triazolium salt 3c $(237 \mathrm{mg}, 77 \%, 2$ steps) as an off-white solid.

${ }^{1}$ H NMR $\left(400 \mathrm{MHz}, \mathrm{CDCl}_{3}\right) \delta 3.18(\mathrm{~d}, 1 \mathrm{H}, J=16.8 \mathrm{~Hz}), 3.34(\mathrm{dd}, 1 \mathrm{H}, J=16.8,4.8 \mathrm{~Hz}), 5.08(\mathrm{~s}$, $2 \mathrm{H}), 5.14$ (dd, $1 \mathrm{H}, J=4.8,4.0 \mathrm{~Hz}), 6.69(\mathrm{~d}, 1 \mathrm{H}, J=4.0 \mathrm{~Hz}), 7.11-7.15(\mathrm{~m}, 2 \mathrm{H}), 7.26-7.33(\mathrm{~m}$, $3 \mathrm{H}), 7.55-7.62(\mathrm{~m}, 1 \mathrm{H}), 7.83-7.86(\mathrm{~m}, 1 \mathrm{H}), 13.3(\mathrm{~s}, 1 \mathrm{H})$;

${ }^{13} \mathbf{C}$ NMR $\left(100 \mathrm{MHz}, \mathrm{CDCl}_{3}\right) \delta 37.5,60.2,62.7,77.7,112.8(\mathrm{dd}, J=18.1,3.3 \mathrm{~Hz}), 113.8(\mathrm{t}, J=$ $14.8 \mathrm{~Hz}), 124.8,125.1,127.8,129.4,133.5(\mathrm{t}, 10.0 \mathrm{~Hz}), 134.9,139.7,146.9,149.8,156.6(\mathrm{dd}, J=$ $258.2,2.5 \mathrm{~Hz})$;

IR (KBr) 3450, 3047, 2914, 1625, 1591, 1541, 1481, 1428, 1245, 1213, 1101, 1018, 966, 791, 741 $\mathrm{cm}^{-1}$;

Anal. Calc'd for $\mathrm{C}_{18} \mathrm{H}_{14} \mathrm{ClF}_{2} \mathrm{~N}_{3} \mathrm{O}$ : C, 59.76; H, 3.90; N, 11.62, Found: C, 59.47; H, 4.16; N, 11.33; Optical Rotation $[\alpha]_{\mathrm{D}}^{21}+207\left(\mathrm{CHCl}_{3}, c 1.00\right)$;

m.p. $237-238^{\circ} \mathrm{C}\left(\right.$ acetone $\left./ \mathrm{H}_{2} \mathrm{O}\right)$.

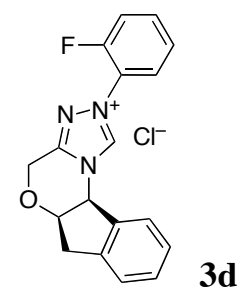

To a solution of iminoether $27(165 \mathrm{mg}, 0.812 \mathrm{mmol})$ in $\mathrm{MeOH}(4.0 \mathrm{~mL})$, 2fluorophenylhydrazine hydrochloride $(132 \mathrm{mg}, 0.812 \mathrm{mmol})$ was added at room temperature. The mixture was warmed to $50{ }^{\circ} \mathrm{C}$ and stirred for $4 \mathrm{~h}$. After cooling to room temperature, the solvents were evaporated under reduced pressure. The residue was washed with $\mathrm{Et}_{2} \mathrm{O}$ and dried in vacuo to afford a corresponding hydrazone hydrochloride. In a flame-dried $20 \mathrm{~mL}$ sealed tube was placed the residue in trimethyl orthoformate $(3.0 \mathrm{~mL})$ and methanol $(1.5 \mathrm{~mL})$. The

[2] Knight, R. L.; Leeper, F. J. J. Chem. Soc. Perkin Trans. 1 1998, 1891-1893. 
reaction mixture was stirred for $29 \mathrm{~h}$ at $80{ }^{\circ} \mathrm{C}$. After the solvents were removed in vacuo, the residue was washed with $\mathrm{Et}_{2} \mathrm{O}$ to give triazolium salt $\mathbf{3 d}$ ( $234 \mathrm{mg}, 84 \%, 2$ steps) as a yellow solid. ${ }^{1} \mathbf{H}$ NMR (400 MHz, $\mathrm{CDCl}_{3}$ ) $\delta 3.11$ (brs, $2 \mathrm{H}$ ), 5.03 (brs, $1 \mathrm{H}$ ), 5.06 (s, $2 \mathrm{H}$ ), 6.62 (brs, $1 \mathrm{H}$ ), $7.22-$ 7.34 (m, 5 H), 7.51-7.56 (m, $1 \mathrm{H}), 7.93-7.95(\mathrm{~m}, 1 \mathrm{H}), 8.01-8.05(\mathrm{~m}, 1 \mathrm{H}), 13.0(\mathrm{~s}, 1 \mathrm{H})$;

${ }^{13} \mathbf{C}$ NMR $\left(100 \mathrm{MHz}, \mathrm{CDCl}_{3}\right) \delta 37.3,60.2,62.4,77.6,117.4(\mathrm{~d}, J=19.0 \mathrm{~Hz}), 123.2(\mathrm{~d}, J=10.7$ $\mathrm{Hz}), 124.9,125.1,125.4(\mathrm{~d}, J=3.3 \mathrm{~Hz}), 125.8,127.7,129.4,132.6$ (d, $J=7.4 \mathrm{~Hz}), 135.0,139.8$, $144.7(\mathrm{~d}, J=5.0 \mathrm{~Hz}), 149.3,154.4(\mathrm{~d}, J=254.9 \mathrm{~Hz})$;

IR (KBr) 3440, 3041, 2922, 1627, 1583, 1537, 1498, 1459, 1259, 1223, 1101, 972, 768, $745 \mathrm{~cm}^{-1}$; Anal. Calc'd for $\mathrm{C}_{18} \mathrm{H}_{15} \mathrm{ClFN}_{3} \mathrm{O}$ : C, 62.89; H, 4.40; N, 12.22, Found: C, 62.60; H, 4.64; N, 11.95;

Optical Rotation $[\alpha]_{\mathrm{D}}^{23}+226\left(\mathrm{CHCl}_{3}, c 1.01\right)$;

m.p. $163-166^{\circ} \mathrm{C}\left(\mathrm{CH}_{3} \mathrm{CN} / \mathrm{Et}_{2} \mathrm{O}\right.$, precipitation).

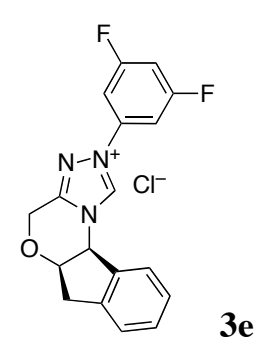

To a solution of iminoether 27 (465 $\mathrm{mg}, 2.28 \mathrm{mmol})$ in $\mathrm{MeOH}(10 \mathrm{~mL}), 3,5-$ difluorophenylhydrazine hydrochloride $(413 \mathrm{mg}, 2.28 \mathrm{mmol})$ was added at room temperature. The mixture was warmed to $50{ }^{\circ} \mathrm{C}$ and stirred for $3 \mathrm{~h}$. After cooling to room temperature, the solvents were evaporated under reduced pressure. The residue was washed with $\mathrm{Et}_{2} \mathrm{O}$ and dried in vacuo to afford a corresponding hydrazone hydrochloride. In a flame-dried $20 \mathrm{~mL}$ sealed tube was placed the residue in trimethyl orthoformate $(4.0 \mathrm{~mL})$ and methanol $(2.0 \mathrm{~mL})$. The reaction mixture was stirred for $24 \mathrm{~h}$ at $80{ }^{\circ} \mathrm{C}$. The solvents were removed in vacuo and the residue was washed with $\mathrm{Et}_{2} \mathrm{O}$ to give triazolium salt $\mathbf{3 e}(730 \mathrm{mg}, 88 \%, 2$ steps) as an off-white solid.

${ }^{1} \mathbf{H}$ NMR (400 MHz, $\left.\mathrm{CDCl}_{3}\right) \delta 3.02(\mathrm{dd}, 1 \mathrm{H}, J=17.2,4.1 \mathrm{~Hz}), 3.10(\mathrm{~d}, 1 \mathrm{H}, 17.2 \mathrm{~Hz}), 4.98(\mathrm{dd}, 1$ H, 4.1, $4.1 \mathrm{~Hz}), 5.06$ (s, $2 \mathrm{H}), 6.41$ (d, $1 \mathrm{H}, J=4.1 \mathrm{~Hz}), 6.87-6.91$ (m, $1 \mathrm{H}), 7.20-7.35$ (m, $3 \mathrm{H})$, 7.97-7.99 (m, $2 \mathrm{H}), 8.07-8.09$ (m, $1 \mathrm{H}), 13.7$ (s, $1 \mathrm{H})$;

${ }^{13}$ C NMR $\left(75 \mathrm{MHz}, \mathrm{CDCl}_{3}\right) \delta 37.1,60.3,62.4,77.5,104.5(\mathrm{~d}, J=30.8 \mathrm{~Hz}), 105.7$ (t, $\left.J=24.8 \mathrm{~Hz}\right)$, $125.0(\mathrm{~d}, J=18.5 \mathrm{~Hz}), 127.7,127.8,129.5,134.6,136.6$ (t, $13.0 \mathrm{~Hz}), 139.9,143.3,149.6,164.7$ (dd, $J=250.4,13.6 \mathrm{~Hz}$ );

IR (KBr) 3440, 3041, 2922, 1627, 1583, 1537, 1498, 1459, 1259, 1223, 1101, 972, 768, $745 \mathrm{~cm}^{-1}$; Anal. Calc'd for $\mathrm{C}_{18} \mathrm{H}_{14} \mathrm{ClF}_{2} \mathrm{~N}_{3} \mathrm{O}$ : C, 59.76; H, 3.90; N, 11.62, Found: C, 59.50; H, 4.16; N, 11.34; Optical Rotation $[\alpha]_{\mathrm{D}}^{23}+214\left(\mathrm{CHCl}_{3}, c 1.02\right)$;

m.p. $212-215^{\circ} \mathrm{C}\left(\mathrm{CH}_{3} \mathrm{CN} / \mathrm{Et}_{2} \mathrm{O}\right.$, precipitation). 


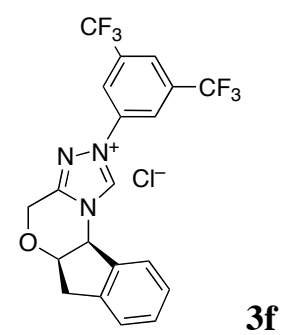

To a solution of iminoether $27(972 \mathrm{mg}, 4.78 \mathrm{mmol})$ in $\mathrm{MeOH}(23 \mathrm{~mL}), 3,5-$ bis(trifluoromethyl)phenylhydrazine hydrochloride ${ }^{[3]}(1.34 \mathrm{~g}, 4.78 \mathrm{mmol})$ was added at room temperature. The mixture was warmed to $50{ }^{\circ} \mathrm{C}$ and stirred for $2 \mathrm{~h}$. After cooling to room temperature, the solvents were evaporated under reduced pressure. The residue was washed with $\mathrm{Et}_{2} \mathrm{O} / \mathrm{hexane}(\mathrm{v} / \mathrm{v}=1: 1)$ and dried in vacuo to afford a corresponding hydrazone hydrochloride. In a flame-dried sealed tube was placed the residue in trimethyl orthoformate $(8.0 \mathrm{~mL})$ and methanol $(4.0 \mathrm{~mL})$. The reaction mixture was stirred for $28 \mathrm{~h}$ at $80{ }^{\circ} \mathrm{C}$. After the solvents were removed in vacuo, the residue was washed with $\mathrm{Et}_{2} \mathrm{O} / \mathrm{CH}_{2} \mathrm{Cl}_{2} / \mathrm{hexane}(\mathrm{v} / \mathrm{v} / \mathrm{v}=2 / 1 / 6)$ to give triazolium salt $\mathbf{3 f}$ (1.33 g, $60 \%, 2$ steps) as a off-white solid.

${ }^{1} \mathbf{H}$ NMR $\left(400 \mathrm{MHz}, \mathrm{CDCl}_{3}\right) \delta 2.78(\mathrm{dd}, 1 \mathrm{H}, J=16.8,4.8 \mathrm{~Hz}), 3.02(\mathrm{~d}, 1 \mathrm{H}, J=16.8 \mathrm{~Hz}), 4.95$ $(\mathrm{dd}, 1 \mathrm{H}, J=4.8,4.0 \mathrm{~Hz}), 5.07(\mathrm{~d}, 1 \mathrm{H}, J=16.0 \mathrm{~Hz}), 5.16(\mathrm{~d}, 1 \mathrm{H}, J=16.0 \mathrm{~Hz}), 6.41(\mathrm{~d}, 1 \mathrm{H}, J=$ 4.0 Hz), 7.14-7.16 (m, $1 \mathrm{H}), 7.25-7.30$ (m, $2 \mathrm{H}), 7.91$ (s, $1 \mathrm{H}), 8.08-8.10(\mathrm{~m}, 1 \mathrm{H}), 8.96(\mathrm{~s}, 2 \mathrm{H})$, $14.0(\mathrm{~s}, 1 \mathrm{H})$

${ }^{13}$ C NMR $\left(100 \mathrm{MHz}, \mathrm{CDCl}_{3}\right) \delta 37.2,60.4,62.6,77.4,120.9(\mathrm{q}, J=3.3 \mathrm{~Hz}), 122.2(\mathrm{q}, J=271 \mathrm{~Hz})$, 123.6 (m), 124.5, 125.2, 127.6, 129.5, 133.5 (q, $J=34.7 \mathrm{~Hz}), 134.4,136.1,139.9$, 144.2, 149.9; IR (KBr) 3415, 2929, 1624, 1591, 1535, 1365, 1281, 1184, 1140, 1074, 901, 741, $683 \mathrm{~cm}^{-1}$; Anal. Calc'd for $\mathrm{C}_{20} \mathrm{H}_{14} \mathrm{ClF}_{6} \mathrm{~N}_{3} \mathrm{O}$ : C, 52.02; H, 3.06; N, 9.10, Found: C, 51.77; H, 3.07; N, 8.84; Optical Rotation $[\alpha]_{\mathrm{D}}^{22}+54.1\left(\mathrm{CHCl}_{3}, c 1.00\right)$;

m.p. $245-248^{\circ} \mathrm{C}$ (acetone/ $\left.\mathrm{H}_{2} \mathrm{O}\right)$.

[3] 3,5-Bistrifluoromethylphenylhydrazine was prepared from 3,5-bistrifluoromethylaniline by the literature procedure, see: Nagarajan, K; Talwalker, P. K.; Kulkarni, C. L.; Venkateswarlu, A; Prabhu, S. S.; Nayak, G. V. Ind. J. Chem. 1984, 23, 1243-1257. 


\section{General Procedure for the preparation of keto-aldehydes 4, 10, 11}

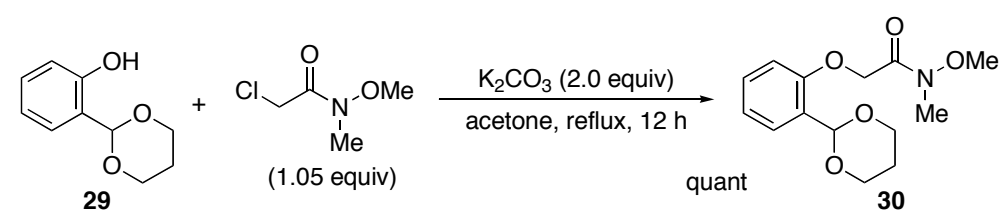

To a mixture of acetal $27^{[4]}(4.37 \mathrm{~g}, 24.2 \mathrm{mmol})$ and $N$-methoxy- $N$-methylchloroacetoamide ${ }^{[5]}$ $(3.50 \mathrm{~g}, 25.4 \mathrm{mmol})$ in acetone $(81 \mathrm{~mL})$ was added $\mathrm{K}_{2} \mathrm{CO}_{3}(6.69 \mathrm{~g}, 48.4 \mathrm{mmol})$. After stirring for $12 \mathrm{~h}$ under reflux, the reaction was cooled to room temperature followed by addition of water. The products were extracted with EtOAc (x3). The combined organic extracts were dried over $\mathrm{Na}_{2} \mathrm{SO}_{4}$ and concentrated under reduced pressure. The residue was purified by silica-gel column chromatography $($ EtOAc/hexane $=4 / 1)$ to give amide $\mathbf{2 8}(6.83$ g, quant) as a colorless solid.

${ }^{1}$ H NMR (400 MHz, $\left.\mathrm{CDCl}_{3}\right) \delta$ 1.40-1.44 (m, $\left.1 \mathrm{H}\right), 2.17-2.29(\mathrm{~m}, 1 \mathrm{H}), 3.23(\mathrm{~s}, 3 \mathrm{H}), 3.72(\mathrm{~s}, 3 \mathrm{H})$, 4.00-4.07 (m, 2 H), 4.21-4.25 (m, 2 H), 4.83 (s, 2 H), 6.01 (s, $1 \mathrm{H}), 6.84$ (d, $1 \mathrm{H}, J=7.6$ Hz), 7.02 (dd, $1 \mathrm{H}, J=8.0,7.6 \mathrm{~Hz}), 7.27$ (ddd, $1 \mathrm{H}, J=8.0,7.6,2.0 \mathrm{~Hz}), 7.64$ (dd, $1 \mathrm{H}, J=7.6,2.0 \mathrm{~Hz}$ );

${ }^{13}$ C NMR (100 MHz, $\left.\mathrm{CDCl}_{3}\right) \delta$ 25.9, 32.3, 61.5, 66.6, 67.5, 96.8, 112.6, 121.7, 127.1, 127.9, 129.7, 155.1, 169.1;

IR (KBr) 2964, 2854, 1685, 1606, 1496, 1458, 1394, 1236, 1178, 1095, 1061, 987, $758 \mathrm{~cm}^{-1}$;

Anal. Calc'd for $\mathrm{C}_{14} \mathrm{H}_{19} \mathrm{NO}_{5}$ : C, 59.78; H, 6.81; N, 4.98; Found: C, 59.76; H, 6.86; N, 4.68;

m.p. $74.0-77.0^{\circ} \mathrm{C}$.

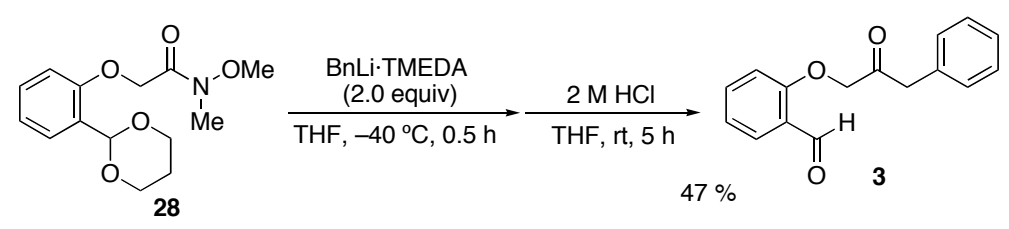

The preparation of keto-aldehyde 3 is representative. To a solution of TMEDA $\left(N, N, N^{\prime}, N^{\prime}-\right.$ tetramethylethylenediamine, $3.11 \mathrm{~mL}, 20.4 \mathrm{mmol})$ in toluene $(7.0 \mathrm{~mL})$ was added $n$-BuLi $(1.6 \mathrm{M}$ in hexane, $12.5 \mathrm{~mL}, 20.0 \mathrm{mmol}$ ) dropwise at room temperature. ${ }^{[6]}$ The resulting solution was heated to $110{ }^{\circ} \mathrm{C}$ and stirred for $30 \mathrm{~min}$. The obtained orange solution of benzyl lithium TMEDA complex was used in the following experiment. To a solution of amide 28 (2.82 $\mathrm{g}$, $10.0 \mathrm{mmol})$ in THF (33 mL) was added benzyl lithium TMEDA complex (2.0 equiv) dropwise at $-40{ }^{\circ} \mathrm{C}$. After stirring for $30 \mathrm{~min}$, the reaction was quenched with saturated $\mathrm{NH}_{4} \mathrm{Cl}$. The products were extracted with EtOAc (x3). The combined organic extracts were dried over $\mathrm{Na}_{2} \mathrm{SO}_{4}$ and concentrated under reduced pressure. After evaporation, the residue was dissolved in THF $(25 \mathrm{ml})$, to which was added $2 \mathrm{M} \mathrm{HCl}(5.0 \mathrm{~mL}, 10 \mathrm{mmol})$ at $0{ }^{\circ} \mathrm{C}$. After stirring for $5 \mathrm{~h}$ at room temperature, the reaction was quenched with saturated $\mathrm{NaHCO}_{3}$. The products were extracted with EtOAc (x3). The combined organic layer was washed with brine, dried over $\mathrm{Na}_{2} \mathrm{SO}_{4}$ and concentrated under reduced pressure. The residue was purified by silica-gel

[4] Gopinath, R.; Haque, S. J.; Patel, B. K. J. Org. Chem. 2002, 67, 5842-5845.

[5] Dolling, U. H.; Frey L. F.; Tillyer, R. D.; Tschaen, D. M. PCT Int. Appl. WO 97/10195, 1997.

[6] Eberhardt, G. G.; Butte, W. A. J. Org. Chem. 1964, 29, 2928-2932. 
column chromatography (EtOAc/hexane $=1 / 4)$ to give keto-aldehyde $\mathbf{3}(1.20 \mathrm{~g}, 47 \%, 2$ steps $)$ as a colorless solid.

${ }^{1}$ H NMR (300 MHz, $\left.\mathrm{CDCl}_{3}\right) \delta 3.92(\mathrm{~s}, 2 \mathrm{H}), 4.75$ (s, $\left.2 \mathrm{H}\right), 6.70$ (d, $\left.1 \mathrm{H}, J=8.4 \mathrm{~Hz}\right), 7.08$ (t, $1 \mathrm{H}$, $J=7.5 \mathrm{~Hz}), 7.22-7.37(\mathrm{~m}, 5 \mathrm{H}), 7.46-7.51(\mathrm{~m}, 1 \mathrm{H}), 7.87$ (dd, $1 \mathrm{H}, J=7.5,1.8 \mathrm{~Hz}), 10.5(\mathrm{~s}, 1 \mathrm{H})$;

${ }^{13} \mathrm{C}$ NMR $\left(75 \mathrm{MHz}, \mathrm{CDCl}_{3}\right) \delta 46.5,72.1,112.3,121.8,125.2,127.5,128.9,129.1,129.4,132.6$, 135.8, 159.7, 189.2, 203.3;

IR (KBr) 3028, 2898, 2863, 2767, 1722, 1685, 1599, 1483, 1284, 1246, 1061, 769, $704 \mathrm{~cm}^{-1}$;

Anal. Calc'd for $\mathrm{C}_{16} \mathrm{H}_{14} \mathrm{O}_{3}$ : C, 75.57; H, 5.55; Found: C, 75.57; H, 5.57;

m.p. $109-110{ }^{\circ} \mathrm{C}$.<smiles>CC(=O)COc1ccccc1C=O</smiles>

10 (Table 3, entry 1)

Prepared according to the general procedure from 28 (1.03 g, $3.64 \mathrm{mmol})$ with MeLi (1.14 M in $\mathrm{Et}_{2} \mathrm{O}, 3.84 \mathrm{~mL}, 4.37 \mathrm{mmol}$ ) followed by hydrolysis with $2 \mathrm{M} \mathrm{HCl}$. The crude product was purified by column chromatography (hexane/EtOAc $=2 / 1$ ) to afford keto-aldehyde 10 (569 mg, $88 \%, 2$ steps) as a colorless oil.

${ }^{1}$ H NMR (300 MHz, $\left.\mathrm{CDCl}_{3}\right) \delta 2.33$ (s, $\left.3 \mathrm{H}\right), 4.69$ (s, $\left.2 \mathrm{H}\right), 6.82(\mathrm{~d}, 1 \mathrm{H}, J=8.5 \mathrm{~Hz}), 7.10$ (dd, 1 $\mathrm{H}, J=7.3,7.3 \mathrm{~Hz}$ ), 7.55 (ddd, $1 \mathrm{H}, J=8.5,7.3,1.7 \mathrm{~Hz}), 7.87$ (dd, $1 \mathrm{H}, J=7.3,1.7 \mathrm{~Hz}), 10.6$ (s, 1 $\mathrm{H})$;

${ }^{13} \mathbf{C}$ NMR $\left(75 \mathrm{MHz}, \mathrm{CDCl}_{3}\right) \delta$ 26.6, 73.0, 112.3, 121.8, 125.1, 129.0, 135.9, 159.8, 189.1, 204.1;

IR (neat) 3419, 3342, 3017, 2904, 2769, 1732, 1685, 1599, 1487, 1394, 1361, 1290, 1241, 1059, $768 \mathrm{~cm}^{-1}$;

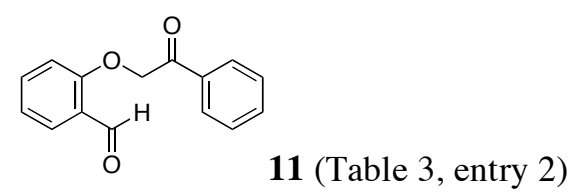

Prepared according to the general procedure from 28 (1.18 g, $4.18 \mathrm{mmol})$ with $\mathrm{PhLi}(0.98 \mathrm{M}$ in cyclohexane/ $\mathrm{Et}_{2} \mathrm{O}, 4.69 \mathrm{~mL}, 4.60 \mathrm{mmol}$ ) followed by hydrolysis with $2 \mathrm{M} \mathrm{HCl}$. The crude product was purified by column chromatography (hexane/EtOAc $=3 / 1$ ) to afford keto-aldehyde 11 (721 $\mathrm{mg}, 72 \%, 2$ steps) as a colorless solid.

${ }^{1}$ H NMR $\left(400 \mathrm{MHz}, \mathrm{CDCl}_{3}\right) \delta 5.43$ (s, $\left.2 \mathrm{H}\right), 6.87(\mathrm{~d}, 1 \mathrm{H}, J=8.4 \mathrm{~Hz}), 7.07(\mathrm{dd}, 1 \mathrm{H}, J=7.2,7.2$ $\mathrm{Hz}), 7.48-7.54$ (m, $3 \mathrm{H}), 7.65$ (tt, $1 \mathrm{H}, J=7.6,1.2 \mathrm{~Hz}$ ), 7.87 (dd, $1 \mathrm{H}, J=8.0,1.6 \mathrm{~Hz}), 7.97-8.00$ (m, $2 \mathrm{H}), 10.6$ (s, $1 \mathrm{H})$;

${ }^{13}$ C NMR (100 MHz, $d_{6}$-acetone) $\delta 71.5,114.6,122.0,126.1,128.3,128.7,129.7,134.6,135.5$, 136.6, 161.7, 189.6, 194.3;

IR (KBr) 3066, 2902, 2875, 2777, 1705, 1685, 1597, 1485, 1288, 1232, 980, $756 \mathrm{~cm}^{-1}$;

Anal. Calc'd for $\mathrm{C}_{15} \mathrm{H}_{12} \mathrm{O}_{3}$ : C, 74.99; H, 5.03; Found: C, 74.96; H, 5.27; 
mp $114-116{ }^{\circ} \mathrm{C}($ EtOAc/hexane $)$.

\section{General Procedure for Benzoin Cyclization of Keto-aldehyde}<smiles>O=C1c2ccccc2OCC1(O)Cc1ccccc1</smiles>

The reaction of keto-aldehyde 4 is representative (Table 2, entry 5). A mixture of 4 (83.5 mg, $0.330 \mathrm{mmol})$ and triazolium salt $3 \mathbf{f}(22.8 \mathrm{mg}, 0.049 \mathrm{mmol})$ in toluene $(1.1 \mathrm{~mL})$ was degassed by two cycles of freeze-pump-thaw. To the mixture was added $\mathrm{Et}_{3} \mathrm{~N}(4.6 \mu \mathrm{L}, 0.033 \mathrm{mmol}$ ) at room temperature under nitrogen atmosphere. After stirring at this temperature for $5 \mathrm{~h}$, the reaction was cooled to $0{ }^{\circ} \mathrm{C}$, and water was added. The products were extracted with EtOAc (x3). The combined organic extracts were dried over $\mathrm{Na}_{2} \mathrm{SO}_{4}$ and concentrated under reduced pressure. The residue was purified by silica-gel column chromatography $\left(\mathrm{EtOAc} / \mathrm{CH}_{2} \mathrm{Cl}_{2} /\right.$ hexane $\left.=1 / 1 / 3\right)$ to give benzoin product $(R)-5^{[7]}(72.6 \mathrm{mg}, 87 \%, 94 \%$ ee) as colorless oil. The enantiomeric excess was assessed by HPLC analysis on a chiral stationary phase: CHIRALPAK ${ }^{\circledR}$ AD-H. (eluent: 2propanol:hexane $=2: 98$, flow rate: $\left.1.0 \mathrm{~mL} / \mathrm{min}, 25{ }^{\circ} \mathrm{C}:(S)-5: \mathrm{t}_{1}=23.8 \mathrm{~min} ;(R)-\mathbf{5}: \mathrm{t}_{2}=28.8 \mathrm{~min}\right)$. The corresponding byproducts 6-9 were also obtained in $11 \%$ combined yield. Since $\beta$ hydroxyketones 6 and 7 were inseparable on silica-gel chromatography $\left(R_{f} 0.2\right.$, silica-gel TLC, EtOAc/hexane $=1: 3$ ), the extent of these side reactions was assessed after treatment with DBU (ca. 1 equiv., THF, room temperature) that converted the aldols into the corresponding enones $\mathbf{8}^{[8]}$ and $\mathbf{9}^{[9]}\left(R_{f} 0.6\right)$.

${ }^{1} \mathbf{H}$ NMR $\left(300 \mathrm{MHz}, \mathrm{CDCl}_{3}\right) \delta 2.97(\mathrm{~d}, 1 \mathrm{H}, J=13.8 \mathrm{~Hz}), 3.04(\mathrm{~d}, 1 \mathrm{H}, J=13.8 \mathrm{~Hz}), 3.58$ (brs, 1 H), 4.11 (d, $1 \mathrm{H}, J=11.3 \mathrm{~Hz}), 4.30$ (d, $1 \mathrm{H}, J=11.3 \mathrm{~Hz}), 7.04-7.33$ (m, $7 \mathrm{H}), 7.54-7.60$ (m, $1 \mathrm{H})$, 7.89 (dd, $1 \mathrm{H}, J=7.8,2.1 \mathrm{~Hz})$;

${ }^{13}$ C NMR (75 MHz, $\left.\mathrm{CDCl}_{3}\right) \delta$ 40.9, 72.2, 73.0, 118.0, 118.4, 122.0, 127.1, 127.6, 128.2, 130.6, 134.3, 136.7, 161.4, 196.0;

IR (neat) 3475, 3062, 3029, 2920, 2871, 1695, 1608, 1477, 1306, 1217, 1093, 1036, 1018, 764, $700 \mathrm{~cm}^{-1}$;

Anal. Calc'd for $\mathrm{C}_{16} \mathrm{H}_{14} \mathrm{O}_{3}$ : C, 75.57; H, 5.55; Found: C, 75.50; H, 5.59;

Optical Rotation $[\alpha]_{\mathrm{D}}{ }^{30}-11.1\left(\mathrm{CHCl}_{3}, c\right.$ 3.04) for $94 \%$ ee; Lit. $^{[7 \mathrm{~b}]}[\alpha]_{\mathrm{D}}{ }^{20}-10.0\left(\mathrm{CHCl}_{3}, c 3.00\right)$ for $91 \%$ ee.

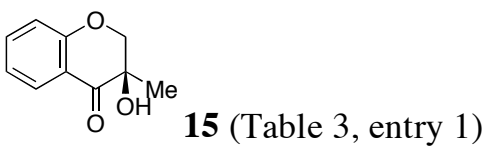

[7] (a) Takikawa, H.; Hachisu, Y.; Bode, J. W.; Suzuki, K. Angew. Chem., Int. Ed. 2006, 45, 3492-3494; (b) Davis, F. A.; Weismiller, M. C. J. Org. Chem. 1990, 55, 3715-3717; (c) Jew, S.-S.; Kim, H.-A.; Kim, J.-H.; Park, H. -G. Heterocycles 1997, 46, 65-70; (d) Kirkiacharian, B. S.; Gomis, M. H.; Tongo, G.; Mahuteau, J.; Brion, J. D. Org. Magn. Reson. 1984, 22, 106-108.

[8] Gill, M. Tetrahedron 1984, 40, 621-626.

[9] Hofmann, H.; Hofmann, P. Justus Liebigs Ann. Chem. 1974, 1301-1314. 
Prepared according to the general procedure from keto-aldehyde 10 (247 mg, $1.38 \mathrm{mmol})$ under the conditions indicated in Table 3. The crude product was purified by column chromatography (hexane/EtOAc $=2 / 3)$ to afford $\alpha$-ketol $15(222 \mathrm{mg}, 90 \%, 94 \%$ ee) as colorless oil. The enantiomeric excess was assessed by HPLC analysis on a chiral stationary phase (CHIRALPAK ${ }^{\circledast}$ AD-H, eluent: 2-propanol:hexane $=2: 98$, flow rate: $1.0 \mathrm{~mL} / \mathrm{min}, 25^{\circ} \mathrm{C}:(R)-15: \mathrm{t}_{1}=17.3 \mathrm{~min} ;(S)$ 15: $\left.\mathrm{t}_{2}=21.5 \mathrm{~min}\right)$.

${ }^{1} \mathbf{H}$ NMR $\left(400 \mathrm{MHz}, \mathrm{CDCl}_{3}\right) \delta 1.47(\mathrm{~s}, 3 \mathrm{H}), 3.61(\mathrm{brs}, 1 \mathrm{H}), 4.21(\mathrm{~d}, 1 \mathrm{H}, J=11.2 \mathrm{~Hz}), 4.31(\mathrm{~d}, 1$ $\mathrm{H}, J=11.2 \mathrm{~Hz}$ ), 6.99 (d, $1 \mathrm{H}, J=8.0 \mathrm{~Hz}), 7.07$ (dd, $1 \mathrm{H}, J=7.8,7.8 \mathrm{~Hz}), 7.53$ (ddd, $1 \mathrm{H}, J=8.0$, 7.8, $1.7 \mathrm{~Hz}), 7.89(\mathrm{dd}, 1 \mathrm{H}, J=7.8,1.7 \mathrm{~Hz})$;

${ }^{13} \mathrm{C}$ NMR $\left(100 \mathrm{MHz}, \mathrm{CDCl}_{3}\right) \delta$ 22.4, 70.6, 74.6, 117.9, 118.1, 121.8, 127.6, 136.5, 161.3, 196.5;

IR (neat) 3471, 3070, 2979, 2929, 2873, 1697, 1608, 1477, 1308, 1215, 1149, 1038, 966, $760 \mathrm{~cm}^{-}$ ;

Anal. Calc'd for $\mathrm{C}_{10} \mathrm{H}_{10} \mathrm{O}_{3}$ : C, 67.41; $\mathrm{H}, 5.66$; Found: $\mathrm{C}, 67.13 ; \mathrm{H}, 5.90$;

Optical Rotation $[\alpha]_{\mathrm{D}}{ }^{23}+15.1\left(\mathrm{CHCl}_{3}, c 1.10\right)$ for $94 \%$ ee; Lit. ${ }^{[7 b]}[\alpha]_{\mathrm{D}}{ }^{20}-9.4\left(\mathrm{CHCl}_{3}, c 1.8\right)$ for $\geq 96 \%$ ee. $^{[10]}$

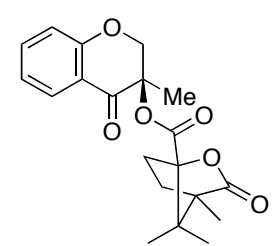

Prepared according to the procedure in our previous report (see reference 7a) from $\alpha$-ketol $15[(S)$ champhanic chloride, DMAP, $\mathrm{CH}_{2} \mathrm{Cl}_{2}$ ].

${ }^{1} \mathbf{H}$ NMR $\left(400 \mathrm{MHz}, \mathrm{CDCl}_{3}\right) \delta 1.03(\mathrm{~s}, 3 \mathrm{H}), 1.08(\mathrm{~s}, 3 \mathrm{H}), 1.12(\mathrm{~s}, 3 \mathrm{H}), 1.68(\mathrm{~s}, 3 \mathrm{H}), 1.70-1.73$ (m, $1 \mathrm{H}), 1.90-1.97(\mathrm{~m}, 1 \mathrm{H}), 2.05-2.11(\mathrm{~m}, 1 \mathrm{H}), 2.41-2.48(\mathrm{~m}, 1 \mathrm{H}), 4.20(\mathrm{~d}, 1 \mathrm{H}, J=11.0 \mathrm{~Hz})$, 5.09 (d, $1 \mathrm{H}, J=11.0 \mathrm{~Hz}), 6.99$ (dd, $1 \mathrm{H}, J=8.3,0.7 \mathrm{~Hz}), 7.09$ (ddd, $1 \mathrm{H}, J=8.0,8.0,0.7 \mathrm{~Hz}$ ), 7.53 (ddd, $1 \mathrm{H}, J=8.3,8.0,1.6 \mathrm{~Hz}), 7.93(\mathrm{dd}, 1 \mathrm{H}, J=8.0,1.6 \mathrm{~Hz}$ );

${ }^{13} \mathbf{C}$ NMR $\left(100 \mathrm{MHz}, \mathrm{CDCl}_{3}\right) \delta 9.7,16.4,16.5,19.8,29.0,30.6,54.6,54.8,71.4,79.2,90.6,117.8$, $119.3,122.2,128.0,136.3,160.4,165.7,177.8,188.8$

IR (KBr) 2976, 2939, 2898, 1788, 1736, 1699, 1606, 1479, 1350, 1313, 1267, 1173, 1088, 760 $\mathrm{cm}^{-1}$;

Anal. Calc'd for $\mathrm{C}_{20} \mathrm{H}_{22} \mathrm{O}_{6}$ : C, 67.03; $\mathrm{H}, 6.19$; Found: $\mathrm{C}, 66.91 ; \mathrm{H}, 6.46$;

Optical Rotation $[\alpha]_{\mathrm{D}}^{29}+9.0\left(\mathrm{CHCl}_{3}, c 1.0\right)$;

mp $188-189^{\circ} \mathrm{C}$ (acetone/hexane).

CCDC 290473 contains the supplementary crystallographic data for this paper. These data can be obtained free of charge via www.ccdc.cam.ac.uk/conts/retrieving.html (or from the Cambridge Crystallographic Data Centre, 12, Union Road, Cambridge CB2 1EZ, UK; fax: (+44) 1223-336033; or deposit@ccdc.cam.ac.uk).

[10] Although the value of opitical rotation indicated the opposite absolute configulation to the reported one, we ensured it by X-ray analysis of the corresponding $(S)$-camphanyl derivative. 


\section{6 (Table 3, entry 2)}

Prepared according to the general procedure from keto-aldehyde 11 (151 $\mathrm{mg}, 0.630 \mathrm{mmol})$ under the conditions indicated in Table 3. The crude product was purified by PTLC $($ hexane/EtOAc $=$ $1 / 3$ ) to afford $\alpha$-ketol 16 (92.6 mg, 61\%, 90\% ee) as a colorless solid. The enantiomeric excess was assessed by HPLC analysis on a chiral stationary phase (CHIRALPAK ${ }^{\circledR}$ AD-H, eluent: 2propanol:hexane $=2: 98$, flow rate: $1.0 \mathrm{~mL} / \mathrm{min}, 25^{\circ} \mathrm{C}:(S)-\mathbf{1 6}: \mathrm{t}_{1}=31.5 \mathrm{~min} ;(R)-16: \mathrm{t}_{2}=39.5$ $\mathrm{min})$. The corresponding benzofuran ${ }^{[11]}$ and aldol were also obtained in $27 \%$ combined yield.

${ }^{1}$ H NMR $\left(400 \mathrm{MHz}, \mathrm{CDCl}_{3}\right) \delta 4.25$ (brs, $\left.1 \mathrm{H}\right), 4.46(\mathrm{~d}, 1 \mathrm{H}, J=11.5 \mathrm{~Hz}), 4.84(\mathrm{~d}, 1 \mathrm{H}, J=11.5$ $\mathrm{Hz}), 6.95(\mathrm{~d}, 1 \mathrm{H}, J=8.4 \mathrm{~Hz}), 7.05(\mathrm{dd}, 1 \mathrm{H}, J=7.8,7.8 \mathrm{~Hz}), 7.27-7.34(\mathrm{~m}, 3 \mathrm{H}), 7.43-7.51(\mathrm{~m}, 3$ $\mathrm{H}), 7.93$ (dd, $1 \mathrm{H}, J=7.6,1.6 \mathrm{~Hz}$ );

${ }^{13}$ C NMR (100 MHz, $\left.\mathrm{CDCl}_{3}\right) \delta$ 73.4, 73.7, 118.0, 119.1, 121.9, 125.9, 127.5, 128.6, 128.7, 136.7, 138.4, 161.4, 194.4;

IR (KBr) 3386, 3059, 3024, 2891, 1689, 1608, 1481, 1460, 1257, 1097, 949, $760 \mathrm{~cm}^{-1}$;

Anal. Calc'd for $\mathrm{C}_{15} \mathrm{H}_{12} \mathrm{O}_{3}$ : C, 74.99; $\mathrm{H}, 5.03$; Found: C, 74.92; H, 5.28;

Optical Rotation $[\alpha]_{\mathrm{D}}^{23}+76.5\left(\mathrm{CHCl}_{3}, c 1.00\right)$ for $90 \%$ ee;

m.p. $83.0-84.0^{\circ} \mathrm{C}($ EtOAc/hexane) .

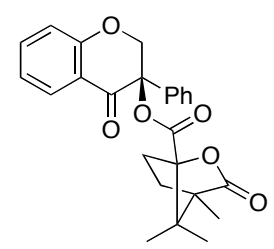

Prepared according to the procedure in our previous report (see reference 7a) from $\alpha$-ketol $16[(S)$ champhanic chloride, DMAP, $\mathrm{CH}_{2} \mathrm{Cl}_{2}$ ].

${ }^{1} \mathbf{H}$ NMR (400 MHz, CDCl $)$ d 1.08 (s, $\left.3 \mathrm{H}\right), 1.11$ (s, 3H), 1.12 (s, $\left.3 \mathrm{H}\right), 1.66-1.73$ (m, $\left.1 \mathrm{H}\right), 1.89$ $1.96(\mathrm{~m}, 1 \mathrm{H}), 2.07-2.14(\mathrm{~m}, 1 \mathrm{H}), 2.43-2.50(\mathrm{~m}, 1 \mathrm{H}), 4.88(\mathrm{~d}, 1 \mathrm{H}, J=11.0 \mathrm{~Hz}), 5.35(\mathrm{~d}, 1 \mathrm{H}, J$ $=11.0 \mathrm{~Hz}), 6.97(\mathrm{~d}, 1 \mathrm{H}, J=8.0 \mathrm{~Hz}), 7.07(\mathrm{dd}, 1 \mathrm{H}, J=8.0,8.0 \mathrm{~Hz}), 7.36-7.41(\mathrm{~m}, 3 \mathrm{H}), 7.50$ (ddd, $1 \mathrm{H}, J=8.0,8.0,1.6 \mathrm{~Hz}), 7.57-7.61(\mathrm{~m}, 2 \mathrm{H}), 7.95(\mathrm{dd}, 1 \mathrm{H}, J=8.0,1.6 \mathrm{~Hz})$;

${ }^{13} \mathbf{C}$ NMR $\left(100 \mathrm{MHz}, \mathrm{CDCl}_{3}\right) \delta 9.8,16.6,16.7,29.0,30.7,54.7,54.9,70.2,81.4,90.8,117.9$, $119.8,122.3,126.6,128.1,128.8,129.5,134.1,136.5,160.3,165.8,177.8,187.1$;

IR (KBr) 3074, 2966, 2873, 1792, 1759, 1701, 1604, 1463, 1265, 1101, 1063, $760 \mathrm{~cm}^{-1}$;

Anal. Calc'd for $\mathrm{C}_{25} \mathrm{H}_{24} \mathrm{O}_{6}$ : C, 71.41; $\mathrm{H}, 5.75$; Found: $\mathrm{C}, 71.22 ; \mathrm{H}, 6.03$;

Optical Rotation $[\alpha]_{\mathrm{D}}^{28}+16.8\left(\mathrm{CHCl}_{3}, c 0.70\right)$;

m.p. $212-214^{\circ} \mathrm{C}$ (acetone/hexane).

CCDC 290475 contains the supplementary crystallographic data for this paper. These data can be obtained free of charge via www.ccdc.cam.ac.uk/conts/retrieving.html (or from the Cambridge Crystallographic Data Centre, 12, Union Road, Cambridge CB2 1EZ, UK; fax: (+44) 1223-336033; ordeposit@ccdc.cam.ac.uk).

[11] Wahab Khan, M.; Jahangir Alam, M.; Rashidb, M. A.; Chowdhuryb, R. Bioorg. Med. Chem. 2005, 13, 4796-4805. 


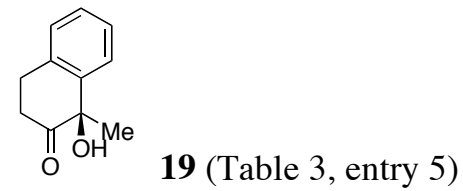

Prepared according to the general procedure from keto-aldehyde 14$^{[12]}$ (134 mg, $\left.0.763 \mathrm{mmol}\right)$ under the conditions indicated in Table 3. The crude product was purified by PTLC (hexane $\left./ \mathrm{CHCl}_{3} / \mathrm{Et}_{2} \mathrm{O}=5 / 3 / 2\right)$ to afford $\alpha$-ketol 19 ${ }^{[7 \mathrm{~b}]}(121 \mathrm{mg}, 90 \%, 60 \%$ ee) as colorless oil. The enantiomeric excess was assessed by HPLC analysis on a chiral stationary phase $\left(\right.$ CHIRALPAK $^{\circledR}$ AD-H, eluent: 2-propanol:hexane $=0.5: 99.5$, flow rate: $1.0 \mathrm{~mL} / \mathrm{min}, 25^{\circ} \mathrm{C}:(S)$ 19: $\left.\mathrm{t}_{1}=13.7 \mathrm{~min} ;(R)-\mathbf{1 9}: \mathrm{t}_{2}=17.3 \mathrm{~min}\right)$.

${ }^{1} \mathbf{H}$ NMR $\left(400 \mathrm{MHz}, \mathrm{CDCl}_{3}\right) \delta 1.56$ (s, $\left.3 \mathrm{H}\right), 2.61-2.69$ (m, $\left.1 \mathrm{H}\right), 2.92-2.99$ (m, $\left.1 \mathrm{H}\right), 3.04-3.11$ (m, 1 H), 3.27-3.35 (m, $1 \mathrm{H}), 3.93$ (s, $1 \mathrm{H}), 7.17$ (brd, $1 \mathrm{H}, J=7.6 \mathrm{~Hz}), 7.23-7.28(\mathrm{~m}, 1 \mathrm{H}), 7.33$ (dd, $1 \mathrm{H}, J=7.6,7.6 \mathrm{~Hz}), 7.66$ (brd, $1 \mathrm{H}, J=7.6 \mathrm{~Hz}$ );

${ }^{13} \mathbf{C}$ NMR $\left(100 \mathrm{MHz} \mathrm{CDCl}_{3}\right) \delta 27.8,27.9,33.5,76.1,125.2,127.4,127.55,127.60,133.6,140.7$, 212.8;

IR (neat) 3483, 3066, 3023, 2975, 2927, 1718, 1458, 1130, 1080, $762 \mathrm{~cm}^{-1}$;

Anal. Calc'd for $\mathrm{C}_{11} \mathrm{H}_{12} \mathrm{O}_{2}$ : C, 74.98; H, 6.86; Found: C, 74.82; H, 6.59;

Optical Rotation $[\alpha]_{\mathrm{D}}{ }^{29}-75.7\left(\mathrm{CHCl}_{3}, c\right.$ 1.14) for $60 \%$ ee; Lit. ${ }^{[7 \mathrm{~b}]}[\alpha]_{\mathrm{D}}{ }^{20}+77.0\left(\mathrm{CHCl}_{3}, c 4.2\right)$ for $76 \%$ ee $(S)$.

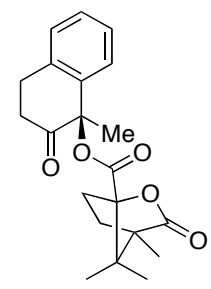

Prepared according to the procedure in our previous report (see reference 7a) from $\alpha$-ketol $16[(S)$ champhanic chloride, DMAP, $\mathrm{CH}_{2} \mathrm{Cl}_{2}$ ].

${ }^{1} \mathbf{H}$ NMR $\left(400 \mathrm{MHz}, \mathrm{CDCl}_{3}\right) \delta 0.95$ (s, $\left.3 \mathrm{H}\right), 1.107$ (s, 3H), 1.111 (s, $\left.3 \mathrm{H}\right), 1.68$ (s, $\left.3 \mathrm{H}\right), 1.70-1.73$ (m, $1 \mathrm{H}), 1.90-1.97$ (m, $1 \mathrm{H}), 2.06-2.13$ (m, $1 \mathrm{H}), 2.43-2.50$ (m, $1 \mathrm{H}), 2.82-2.86$ (m, $2 \mathrm{H}), 3.06-$ 3.13 (m, $1 \mathrm{H}), 3.25-3.33$ (m, $1 \mathrm{H}), 7.19-7.31$ (m, $4 \mathrm{H})$;

${ }^{13} \mathbf{C}$ NMR $\left(100 \mathrm{MHz}, \mathrm{CDCl}_{3}\right) \delta$ 9.7, 16.3, 16.7, 27.1, 28.1, 29.1, 30.6, 37.1, 54.6, 54.8, 80.9, 90.4, $124.5,127.2,127.9,128.0,135.2,138.4,165.6,177.8,206.1$;

IR (KBr) 2975, 2939, 2873, 1785, 1751, 1738, 1491, 1446, 1315, $12751107,1068,1022,769 \mathrm{~cm}^{-}$ ${ }^{1}$;

Anal. Calc'd for $\mathrm{C}_{21} \mathrm{H}_{24} \mathrm{O}_{5}$ : C, 70.77; $\mathrm{H}, 6.79$, Found: C, 70.53; H, 6.84;

Optical Rotation $[\alpha]_{\mathrm{D}}{ }^{30}+13.6\left(\mathrm{CHCl}_{3}, c 0.93\right)$;

m.p. $212-214{ }^{\circ} \mathrm{C}$ (acetone/hexane).

CCDC 290478 contains the supplementary crystallographic data for this paper. These data can be obtained free of charge via www.ccdc.cam.ac.uk/conts/retrieving.html (or from the Cambridge Crystallographic Data Centre, 12, Union Road, Cambridge CB2 1EZ, UK; fax: (+44) 1223-336033; or deposit@ccdc.cam.ac.uk).

[12] Enders, D.; Niemeier, O. Synlett 2004, 2111-2114. 


\section{Preparation of keto-aldehydes 2 (Scheme 2)}<smiles>Oc1ccc(C2OCCCO2)c(O)c1</smiles>

To a solution of aldehyde $20(3.81 \mathrm{~g}, 25.0 \mathrm{mmol})$ in pyridine $(5 \mathrm{~mL})$ was added $\mathrm{Ac}_{2} \mathrm{O}(5 \mathrm{~mL})$ at $0{ }^{\circ} \mathrm{C}$. The reaction mixture was allowed to warm to room temperature and stirred for $4 \mathrm{~h}$. After dilution with water at $0{ }^{\circ} \mathrm{C}$, the products were extracted with $\mathrm{Et}_{2} \mathrm{O}(\mathrm{x} 3)$. The combined organic layer was washed with $1 \mathrm{M} \mathrm{HCl}(\mathrm{x} 2), \mathrm{H}_{2} \mathrm{O}$ and brine followed by drying over $\mathrm{Na}_{2} \mathrm{SO}_{4}$. After the solvents were evaporated under reduced pressure, the residue was dissolved in 1,3propanediol $(7.3 \mathrm{~mL})$ and triethylorthoformate $(8.3 \mathrm{~mL})$. To the mixture was added tetrabutylammonium tribromide $(241 \mathrm{mg}, 0.500 \mathrm{mmol})$ at $10^{\circ} \mathrm{C}$. After stirring for $1 \mathrm{~h}$ at room temperature, $\mathrm{MeOH}(5 \mathrm{~mL})$ and $\mathrm{K}_{2} \mathrm{CO}_{3}(3.46 \mathrm{~g}, 25.0 \mathrm{mmol})$ were added to the mixture at $0{ }^{\circ} \mathrm{C}$. The resulting suspension was allowed to warm to room temperature and stirred for $1 \mathrm{~h}$. Extraction with EtOAc (x3) followed by washing with brine, drying over $\mathrm{Na}_{2} \mathrm{SO}_{4}$ and purification with column chromatography (hexane/EtOAc $=3 / 1$ ) gave acetal 21 [5.00 g, 95\% (2 steps)] as colorless oil.

${ }^{1} \mathbf{H}$ NMR $\left(400 \mathrm{MHz}, \mathrm{CDCl}_{3}\right) \delta 1.45-1.50(\mathrm{~m}, 1 \mathrm{H}), 2.17-2.29$ (m, $\left.1 \mathrm{H}\right), 3.75(\mathrm{~s}, 3 \mathrm{H}), 3.94-4.01$ (m, $2 \mathrm{H}), 4.24-4.29$ (m, $2 \mathrm{H}), 5.58(\mathrm{~s}, 1 \mathrm{H}), 6.41(\mathrm{dd}, 1 \mathrm{H}, J=8.4,2.4 \mathrm{~Hz}), 6.44(\mathrm{~d}, 1 \mathrm{H}, J=2.4$ $\mathrm{Hz}), 7.05$ (d, $1 \mathrm{H}, J=8.4 \mathrm{~Hz}), 7.93(\mathrm{~s}, 1 \mathrm{H})$;

${ }^{13}$ C NMR (100 MHz, $\left.\mathrm{CDCl}_{3}\right) \delta$ 25.7, 55.2, 67.3, 102.1, 103.0, 105.8, 114.9, 128.5, 156.4, 161.2;

IR (neat) 3369, 2962, 2861, 1631, 1588, 1513, 1395, 1274, 1204, 1156, 1107, 1093, $984 \mathrm{~cm}^{-1}$; Anal. Calc'd for $\mathrm{C}_{11} \mathrm{H}_{14} \mathrm{O}_{4}$ : C, 62.85; H, 6.71; Found: C, 63.06; H, 6.50 .<smiles>COc1ccc(C2OCCO2)c(OCC(=O)N(C)OC)c1</smiles>

To a mixture of acetal $21(4.10 \mathrm{~g}, 19.5 \mathrm{mmol})$ and $N$-methoxy- $N$-methylchloroacetoamide ${ }^{[13]}(2.82$ $\mathrm{g}, 20.5 \mathrm{mmol})$ in acetone $(65 \mathrm{~mL})$ was added $\mathrm{K}_{2} \mathrm{CO}_{3}(5.39 \mathrm{~g}, 20.5 \mathrm{mmol})$. After stirring for 14 $\mathrm{h}$ under reflux, the reaction was cooled to room temperature followed by addition of water. The aqueous layer was extracted with EtOAc (x3). The combined organic layer was washed with brine, dried over $\mathrm{Na}_{2} \mathrm{SO}_{4}$, and concentrated under reduced pressure. The residue was purified by silica-gel column chromatography (EtOAc/ hexane $=3 / 2$ to $1 / 0)$ to give amide $22(6.08 \mathrm{~g}$, quant) as a colorless amorphous solid.

${ }^{1} \mathbf{H}$ NMR (400 MHz, $\left.\mathrm{CDCl}_{3}\right) \delta$ 1.38-1.43 (m, $\left.1 \mathrm{H}\right), 2.15-2.28(\mathrm{~m}, 1 \mathrm{H}), 3.23(\mathrm{~s}, 3 \mathrm{H}), 3.73(\mathrm{~s}, 3 \mathrm{H})$, 3.78 (s, 3 H), 3.98-4.05 (m, 2 H), 4.20-4.24 (m, 2 H), 4.80 (s, 2 H), 5.93 (s, 1 H), 6.41 (d, 1 H, J= $2.0 \mathrm{~Hz}$ ), 6.55 (dd, $1 \mathrm{H}, J=8.4,2.0 \mathrm{~Hz}), 7.55$ (d, $1 \mathrm{H}, J=8.4$ );

${ }^{13} \mathbf{C}$ NMR $\left(100 \mathrm{MHz}, \mathrm{CDCl}_{3}\right) \delta 25.9,32.4,55.4,61.6,66.6,67.5,96.8,100.1,105.7,120.9,128.0$, $156.2,160.8,169.0$

IR (ATR) 2959, 2885, 2838, 1673, 1617, 1590, 1513, 1451, 1396, 1258, 1092, 1051, 983, 829,

[13] Dolling, U. H.; Frey L. F.; Tillyer, R. D.; Tschaen, D. M. PCT Int. Appl. WO 97/10195, 1997. 
$794 \mathrm{~cm}^{-1}$

Anal. Calc'd for $\mathrm{C}_{15} \mathrm{H}_{21} \mathrm{NO}_{6}$ : C, 57.87; H, 6.80; N, 4.77; Found: $\mathrm{C}, 57.64 ; \mathrm{H}, 6.94 ; \mathrm{N}, 4.77$;<smiles>COc1ccc(C=O)c(OCC(=O)Cc2ccc(OC)c(OC)c2)c1</smiles>

To a solution of amide $(7.82 \mathrm{~g}, 25.0 \mathrm{mmol})$ in THF $(50 \mathrm{~mL})$ was added 3,4dimethoxyphenylmethylmagnesium chloride ${ }^{[14]}$ [generated from 3,4-dimethoxybenzyl chloride (9.78 g, $37.6 \mathrm{mmol}), \mathrm{Mg}(6.09 \mathrm{~g}, 250 \mathrm{mmol})$ and 1,2-dibromoethane (2 drops) in THF (100 mL) at $-10{ }^{\circ} \mathrm{C}$ ] dropwise at $-20{ }^{\circ} \mathrm{C}$ by steel cannula. After $15 \mathrm{~min}$ at this temperature, the reaction was quenched with saturated $\mathrm{NH}_{4} \mathrm{Cl}$. The products were extracted with EtOAc (x3). The combined organic layer was concentrated under reduced pressure. After evaporation, the residue was purified by silica-gel column chromatography (EtOAc/ hexane $=1 / 2$ to $1 / 1$ ) to give the corresponding keto-acetal (10.8 g, containing some impurities). Keto-acetal was dissolved in THF $(25 \mathrm{~mL})$, to which was added $2 \mathrm{M} \mathrm{HCl}(5.0 \mathrm{~mL})$ at $0{ }^{\circ} \mathrm{C}$. After stirring for $2 \mathrm{~h}$ at this temperature, the reaction was quenched with saturated $\mathrm{NaHCO}_{3}$. The products were extracted with EtOAc (x3). The combined organic layer was washed with brine, dried over $\mathrm{Na}_{2} \mathrm{SO}_{4}$ and concentrated under reduced pressure. The residue was washed with $\mathrm{Et}_{2} \mathrm{O}$ to give keto-aldehyde $2(7.53 \mathrm{~g}, 87 \%)$ as a colorless solid.

${ }^{1}$ H NMR (400 MHz, $\mathrm{CDCl}_{3}$ ) $\delta 3.79$ (s, $\left.3 \mathrm{H}\right), 3.84$ (s, $\left.3 \mathrm{H}\right), 3.855$ (s, $\left.2 \mathrm{H}\right), 3.863$ (s, $\left.3 \mathrm{H}\right), 4.71$ (s, 2 H), $6.15(\mathrm{~d}, 1 \mathrm{H}, J=2.2 \mathrm{~Hz}), 6.58(\mathrm{dd}, 1 \mathrm{H}, J=8.8,2.2 \mathrm{~Hz}), 6.74(\mathrm{~d}, 1 \mathrm{H}, J=1.6 \mathrm{~Hz}), 6.78(\mathrm{dd}, 1$ $\mathrm{H}, J=8.4,1.6 \mathrm{~Hz}), 6.83$ (d, $1 \mathrm{H}, J=8.4 \mathrm{~Hz}), 7.83(\mathrm{~d}, 1 \mathrm{H}, J=8.8 \mathrm{~Hz}), 10.4(\mathrm{~s}, 1 \mathrm{H})$;

${ }^{13} \mathbf{C}$ NMR $\left(100 \mathrm{MHz}, \mathrm{CDCl}_{3}\right) \delta 46.1,55.6,55.8,55.9,71.8,98.7,106.6,111.4,112.2,119.0$, 121.5, 124.8, 131.2, 148.3, 149.1, 161.2, 165.8, 187.6, 203.4;

IR (KBr) 3023, 2940, 2904, 2880, 2838, 1727, 1678, 1599, 1514, 1261, 1138, 1023, 962, 750, 725 $\mathrm{cm}^{-1}$;

Anal. Calc'd for $\mathrm{C}_{19} \mathrm{H}_{20} \mathrm{O}_{6}$ : C, 66.27; $\mathrm{H}, 5.85$; Found: $\mathrm{C}, 66.03 ; \mathrm{H}, 5.87$;

m.p. $117-118^{\circ} \mathrm{C}$ (acetone/hexane).

[14] Hashigaki, K.; Kan, K.; Qais, N.; Takeuchi, Y.; Yamato, M. Chem. Pharm. Bull. 1991, 39, $1126-1131$. 


\section{Synthesis of (+)-sappanone B (Scheme 3)}<smiles>COc1ccc2c(c1)OCC(O)(c1ccc(OC)c(OC)c1)C2=O</smiles>

A mixture of $2(6.22 \mathrm{~g}, 18.1 \mathrm{mmol})$ and triazolium salt $3 \mathbf{f}(626 \mathrm{mg}, 1.36 \mathrm{mmol})$ in toluene $(90 \mathrm{~mL})$ was degassed by two cycles of freeze-pump-thaw. To the mixture was added $\mathrm{Et}_{3} \mathrm{~N}(189 \mu \mathrm{L}$, $1.36 \mathrm{mmol}$ ) at room temperature under nitrogen atmosphere. After stirring at this temperature for $12 \mathrm{~h}$, the reaction was cooled to $0{ }^{\circ} \mathrm{C}$ followed by the addition of water. The products were extracted with EtOAc (x3). The combined organic extracts were dried over $\mathrm{Na}_{2} \mathrm{SO}_{4}$ and concentrated under reduced pressure. The residue was purified by silica-gel column chromatography $($ EtOAc/hexane $=1 / 2)$ to give benzoin product $(R)-\mathbf{2 3}(5.73 \mathrm{~g}, 92 \%, 95 \%$ ee$)$ as light-yellow oil. The enantiomeric excess was assessed by HPLC analysis on a chiral stationary phase: CHIRALPAK ${ }^{\circledR}$ IA (eluent: 2-propanol:hexane $=40: 60$, flow rate: $1.0 \mathrm{~mL} / \mathrm{min}, 25{ }^{\circ} \mathrm{C}:(S)$ 23: $\left.\mathrm{t}_{1}=9.0 \mathrm{~min} ;(R)-\mathbf{2 3}: \mathrm{t}_{2}=16.2 \mathrm{~min}\right)$.

${ }^{1} \mathbf{H}$ NMR $\left(400 \mathrm{MHz}, \mathrm{CDCl}_{3}\right) \delta 2.90(\mathrm{~d}, 1 \mathrm{H}, J=14.4 \mathrm{~Hz}), 2.95$ (d, $\left.1 \mathrm{H}, J=14.4 \mathrm{~Hz}\right), 3.65$ (brs, 1 H), 3.85 (s, $3 \mathrm{H}), 3.86(\mathrm{~s}, 3 \mathrm{H}), 3.88(\mathrm{~s}, 3 \mathrm{H}), 4.09$ (d, $1 \mathrm{H}, J=10.8 \mathrm{~Hz}), 4.29$ (d, $1 \mathrm{H}, J=10.8 \mathrm{~Hz})$, $6.48(\mathrm{~d}, 1 \mathrm{H}, J=2.4 \mathrm{~Hz}), 6.66(\mathrm{dd}, 1 \mathrm{H}, J=8.8,2.4 \mathrm{~Hz}), 6.71-6.74(\mathrm{~m}, 2 \mathrm{H}), 6.79$ (brd, $1 \mathrm{H}, J=$ $8.4 \mathrm{~Hz}), 7.81(\mathrm{~d}, 1 \mathrm{H}, J=8.8 \mathrm{~Hz})$;

${ }^{13} \mathrm{C}$ NMR $\left(100 \mathrm{MHz}, \mathrm{CDCl}_{3}\right) \delta$ 40.8, 55.7, 55.8 (2 C), 72.4, 72.7, 100.8, 110.7, 110.8, 112.0, $113.7,122.5,126.9,129.0,148.0,148.4,163.4,166.5,194.4$;

IR (neat) 3475, 3062, 3029, 2920, 2871, 1695, 1608, 1477, 1306, 1217, 1093, 1036, 1018, 764, $700 \mathrm{~cm}^{-1}$;

Anal. Calc'd for $\mathrm{C}_{19} \mathrm{H}_{20} \mathrm{O}_{6}$ : C, 66.27; H, 5.85; Found: C, 66.49; H, 5.61;

Optical Rotation $[\alpha]_{\mathrm{D}}{ }^{33}-29.7\left(\mathrm{CHCl}_{3}, c 1.01\right)$ for $95 \%$ ee; Lit. $(1)^{[15]}[\alpha]_{\mathrm{D}}{ }^{20}-30.2\left(\mathrm{CHCl}_{3}, c 1.68\right)$ for $94 \%$ ee; (2) $[\alpha]_{\mathrm{D}}{ }^{20}-32.1\left(\mathrm{CHCl}_{3}, c 1.00\right)$.<smiles></smiles>

$\mathrm{NaSC}_{12} \mathrm{H}_{25}$ was prepared by the following method. To a solution of dodecanethiol in $\mathrm{MeOH}$ was added sodium methoxide ( $28 \mathrm{wt} \%$ in $\mathrm{MeOH}, 1$ equiv). After the solvents were removed by evaporation, the residue was dried at $50{ }^{\circ} \mathrm{C}$ overnight to give $\mathrm{NaSC}_{12} \mathrm{H}_{25}$ as a white solid, which was used in the following experiment.

To a solution of $\alpha$-ketol 23 (141 mg, $0.410 \mathrm{mmol})$ in DMF (2.0 mL) was added $\mathrm{NaSC}_{12} \mathrm{H}_{25}(552$ $\mathrm{mg}, 2.46 \mathrm{mmol}$ ) at room temperature. The reaction was heated to $80{ }^{\circ} \mathrm{C}$ and stirred for $5 \mathrm{~h}$ at this temperature. The reaction was quenched with saturated $\mathrm{NH}_{4} \mathrm{Cl}$ at room temperature, and products were extracted with EtOAc (x3). The combined organic extracts were washed with brine, dried over $\mathrm{Na}_{2} \mathrm{SO}_{4}$ and concentrated under reduced pressure. The residue was purified by silica-gel column chromatography (EtOAc/hexane = 1/2 to 3/2) to give phenol $24(124 \mathrm{mg}, 92 \%)$ as a colorless solid.

[15] (a) Davis, F. A.; Chen, B.-C. J. Org. Chem. 1993, 58, 1751-1753; (b) Namikoshi, M.; Saitoh, T. Chem. Pharm. Bull. 1987, 35, 3597-3602. 
${ }^{1} \mathbf{H}$ NMR $\left(400 \mathrm{MHz}, \mathrm{CDCl}_{3}\right) \delta 2.92(\mathrm{~d}, 1 \mathrm{H}, J=14.4 \mathrm{~Hz}), 2.97(\mathrm{~d}, 1 \mathrm{H}, J=14.4 \mathrm{~Hz}), 3.74$ (brs, 1 H), $3.836(\mathrm{~s}, 3 \mathrm{H}), 3.842(\mathrm{~s}, 3 \mathrm{H}), 4.10(\mathrm{~d}, 1 \mathrm{H}, J=11.2 \mathrm{~Hz}), 4.28(\mathrm{~d}, 1 \mathrm{H}, J=11.2 \mathrm{~Hz}), 6.45(\mathrm{~d}, 1$ $\mathrm{H}, J=2.0 \mathrm{~Hz}), 6.58(\mathrm{dd}, 1 \mathrm{H}, J=8.8,2.0 \mathrm{~Hz}), 6.72-6.75(\mathrm{~m}, 2 \mathrm{H}), 6.80$ (brd, $1 \mathrm{H}, J=8.4 \mathrm{~Hz})$, 7.40 (brs, $1 \mathrm{H}), 7.77$ (d, $1 \mathrm{H}, J=8.8 \mathrm{~Hz}$ );

${ }^{13}$ C NMR (100 MHz, $\left.\mathrm{CDCl}_{3}\right) \delta$ 40.8, 55.8 (2 C), 72.2, 72.8, 103.3, 111.0, 111.3, 111.9, 113.7, 122.7, 126.9, 129.6, 147.9, 148.3, 163.4, 164.0, 194.6;

IR (ATR) 3371, 3266, 2940, 2833, 1672, 1611, 1584, 1514, 1475, 1455, 1264, 1160, 1029, 952 $\mathrm{cm}^{-1}$;

Anal. Calc'd for $\mathrm{C}_{18} \mathrm{H}_{18} \mathrm{O}_{6}$ : C, 65.45; H, 5.49; Found: C, 65.69; H, 5.74;

Optical Rotation $[\alpha]_{\mathrm{D}}^{23}-28.2\left(\mathrm{CHCl}_{3}, c 1.01\right)$ for $95 \%$ ee.

m.p. $116-117^{\circ} \mathrm{C}$.

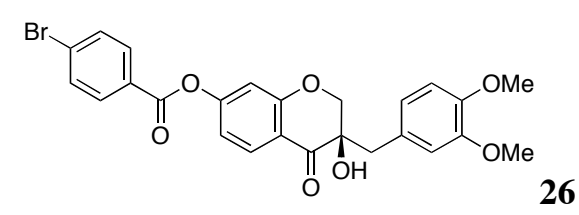

${ }^{1} \mathbf{H}$ NMR $\left(400 \mathrm{MHz}, \mathrm{CDCl}_{3}\right) \delta 2.95(\mathrm{~d}, 1 \mathrm{H}, J=14.4 \mathrm{~Hz}), 3.00(\mathrm{~d}, 1 \mathrm{H}, J=14.4 \mathrm{~Hz}), 3.58$ (brs, 1 H), $3.86(\mathrm{~s}, 3 \mathrm{H}), 3.87$ (s, $3 \mathrm{H}), 4.16(\mathrm{~d}, 1 \mathrm{H}, J=11.5 \mathrm{~Hz}), 4.34$ (d, $1 \mathrm{H}, J=11.5 \mathrm{~Hz}), 6.72-6.74$ (m, $2 \mathrm{H}), 6.81$ (brd, $1 \mathrm{H}, J=8.8 \mathrm{~Hz}$ ), 6.97-7.00 (m, $2 \mathrm{H}), 7.68$ (brd, $2 \mathrm{H}, J=8.8 \mathrm{~Hz}$ ), 7.96 (brd, 1 $\mathrm{H}, J=8.8 \mathrm{~Hz}), 8.05$ (brd, $2 \mathrm{H}, J=8.8 \mathrm{~Hz}$ );

${ }^{13}$ C NMR (100 MHz, $\left.\mathrm{CDCl}_{3}\right) \delta$ 40.6, 55.8 (2 C), 72.6, 73.1, 111.0, 111.2, 113.7, 116.2, 116.5, $122.7,126.6,127.7,129.0,129.5,131.7,132.2,148.2,148.6,157.1,162.5,1636,195.0$;

IR (ATR) 3448, 3098, 2999, 2957, 2832, 1734, 1682, 1609, 1588, 1514, 1431, 1260, 1221, 1139 , 1024, 1009, $748 \mathrm{~cm}^{-1}$;

Anal. Calc'd for $\mathrm{C}_{25} \mathrm{H}_{21} \mathrm{BrO}_{7}$ : C, 58.49; $\mathrm{H}, 4.12$; Found: C, 58.53; H, 4.24;

Optical Rotation $[\alpha]_{\mathrm{D}}{ }^{26}-5.2\left(\mathrm{CHCl}_{3}, c 1.0\right)$.

m.p. $145-146.5^{\circ} \mathrm{C}$ (acetone/hexane).

CCDC 627134 contains the supplementary crystallographic data for this paper. These data can be obtained free of charge via www.ccdc.cam.ac.uk/conts/retrieving.html (or from the Cambridge Crystallographic Data Centre, 12, Union Road, Cambridge CB2 1EZ, UK; fax: (+44) 1223-336033; or deposit@ccdc.cam.ac.uk).<smiles>O=C1c2ccc(O)cc2OC[C@]1(O)Cc1ccc(O)c(O)c1</smiles>

To a solution of phenol $24(1.02 \mathrm{~g}, 3.07 \mathrm{mmol})$ in $\mathrm{CH}_{2} \mathrm{Cl}_{2}(70 \mathrm{~mL})$ was added $\mathrm{BBr}_{3}(2.38 \mathrm{~g}, 9.50$ mmol) in $\mathrm{CH}_{2} \mathrm{Cl}_{2}(30 \mathrm{~mL})$ dropwise at $-10{ }^{\circ} \mathrm{C}$. The reaction was allowed to warm to $0{ }^{\circ} \mathrm{C}$ and stirred for $30 \mathrm{~min}$ at this temperature. After the reaction was quenched with $\mathrm{H}_{2} \mathrm{O}$, the products were extracted with EtOAc (x3). The combined organic extracts were concentrated under reduced pressure. The residue was purified by column chromatography on oxalated silica- 
gel $^{[16]}$ [under an argon atmosphere, eluent: $\mathrm{Et}_{2} \mathrm{O} /$ pentane $=1 / 1$ (degassed)] followed by drying in vacuo overnight to give (+)-sappanone B (1, ca. $790 \mathrm{mg}$, ca. 88\%, containing $\left.\mathrm{Et}_{2} \mathrm{O}\right)$ as an offwhite solid. Although an irremovable $\mathrm{Et}_{2} \mathrm{O}$ was contained in the final product, ${ }^{[17]}$ it was replaceable with other solvents (acetone, $\mathrm{MeOH}$ ) by co-evaporation. The ${ }^{1} \mathrm{H}$ NMR and opitical rotation was measured after co-evaporation with $d_{6}$-acetone and $\mathrm{MeOH}$, respectively.

${ }^{1} \mathbf{H}$ NMR (400 MHz, $d_{6}$-acetone) $\delta 2.77(\mathrm{~d}, 1 \mathrm{H}, J=13.9 \mathrm{~Hz}), 2.85(\mathrm{~d}, 1 \mathrm{H}, J=13.9 \mathrm{~Hz}), 4.03(\mathrm{~d}$, $1 \mathrm{H}, J=11.5 \mathrm{~Hz}), 4.17(\mathrm{~d}, 1 \mathrm{H}, J=11.5 \mathrm{~Hz}), 4.44(\mathrm{~s}, 1 \mathrm{H}), 6.44(\mathrm{~d}, 1 \mathrm{H}, J=2.2 \mathrm{~Hz}), 6.58(\mathrm{dd}, 1 \mathrm{H}$, $J=8.1,2.0 \mathrm{~Hz}), 6.63(\mathrm{dd}, 1 \mathrm{H}, J=8.6,2.2 \mathrm{~Hz}), 6.73(\mathrm{~d}, 1 \mathrm{H}, J=8.1 \mathrm{~Hz}) .6 .78(\mathrm{~d}, 1 \mathrm{H}, J=2.0 \mathrm{~Hz})$, 7.72 (d, $1 \mathrm{H}, J=8.6 \mathrm{~Hz}$ ), 7.72 (brs, $2 \mathrm{H}$ ), 9.53 (brs, $1 \mathrm{H}$ );

${ }^{13} \mathbf{C}$ NMR (100 MHz, $d_{6}$-DMSO) $\delta 15.2\left(\mathrm{Et}_{2} \mathrm{O}\right), 38.9$ (conceiled by $d_{6}$-DMSO), $64.9\left(\mathrm{Et}_{2} \mathrm{O}\right), 72.0$, 72.2, 102.3, 111.0, 111.8, 115.0, 118.1, 121.5, 126.1, 129.2, 143.9, 144.6, 162.7, 164.6, 193.0;

IR (ATR) 3314, 2976, 2926, 2873, 1668, 1600, 1580, 1519, 1473, 1418, 1366, 1276, 1237, 1083, $1031,954 \mathrm{~cm}^{-1}$;

HRMS (EI-MS) calc'd for $\mathrm{C}_{16} \mathrm{H}_{14} \mathrm{O}_{6}$ : $\left[\mathrm{M}^{+}\right]$302.0788; Found: 302.0782;

Optical Rotation $[\alpha]_{\mathrm{D}}{ }^{25}+51(\mathrm{MeOH}, c 1.0)$ for $95 \%$ ee; Lit. $(1)^{[18 a]}[\alpha]_{\mathrm{D}}{ }^{24}+51.6(\mathrm{MeOH}, c 1.00)$; (2) $[\alpha]_{\mathrm{D}}^{25}+53.7(\mathrm{MeOH}, c 0.41){ }^{[18 b]}$<smiles>COc1ccc2c(c1)OCC(O)(c1ccc(OC)c(OC)c1)C2O</smiles>

To a solution of $\mathbf{1}(127 \mathrm{mg}, 0.420 \mathrm{mmol})$ in pyridine $(0.8 \mathrm{~mL})$ was added dropwise $\mathrm{Ac}_{2} \mathrm{O}(0.4 \mathrm{~mL})$ at $0{ }^{\circ} \mathrm{C}$. The reaction mixture was stirred for $1 \mathrm{~h}$ at this temperature. After dilution with water, the products were extracted with $\mathrm{Et}_{2} \mathrm{O}(\mathrm{x} 3)$. The combined organic extracts were washed with $1 \mathrm{M} \mathrm{HCl}(\mathrm{x} 2), \mathrm{H}_{2} \mathrm{O}$ and brine followed by drying over $\mathrm{MgSO}_{4}$. After concentration under reduced pressure, the residue was purified by PTLC (hexane/EtOAc $=2 / 3$ ) to give $O$ triacetylsappanone B (25) (166 mg, 92\%, 95\% ee) as a colorless amorphous solid. The enantiomeric excess was assessed by HPLC analysis on a chiral stationary phase: CHIRALPAK ${ }^{\circledR}$ IA (eluent: 2-propanol:hexane $=50: 50$, flow rate: $1.0 \mathrm{~mL} / \mathrm{min}, 25{ }^{\circ} \mathrm{C}:(S)-25: \mathrm{t}_{1}=9.4 \mathrm{~min} ;(R)-25$ : $\left.\mathrm{t}_{2}=12.5 \mathrm{~min}\right)$.

${ }^{1}$ H NMR (400 MHz, $\left.\mathrm{CDCl}_{3}\right) \delta 2.29$ (s, $\left.6 \mathrm{H}\right), 2.34(\mathrm{~s}, 3 \mathrm{H}), 2.89(\mathrm{~d}, 1 \mathrm{H}, J=14.2 \mathrm{~Hz}), 3.02(\mathrm{~d}, 1 \mathrm{H}$, $J=14.2 \mathrm{~Hz}$ ), 3.61 (brs, $1 \mathrm{H}), 4.10(\mathrm{~d}, 1 \mathrm{H}, J=11.5 \mathrm{~Hz}), 4.30(\mathrm{~d}, 1 \mathrm{H}, J=11.5 \mathrm{~Hz}), 6.85-6.88(\mathrm{~m}$, $2 \mathrm{H}), 7.08-7.14(\mathrm{~m}, 3 \mathrm{H}), 7.92(\mathrm{~d}, 1 \mathrm{H}, J=8.6 \mathrm{~Hz})$;

${ }^{13} \mathrm{C}$ NMR (100 MHz, $\mathrm{CDCl}_{3}$ ) $\delta 20.6$ (2 C), 21.1, 40.1, 72.1, 72.7, 111.1, 116.0, 116.3, 123.0, 125.5, 128.7, 129.0, 133.0, 141.2, 141.6, 157.1, 162.4, 168.16, 168.19, 168.4, 194.7;

IR (ATR) 3478, 2935, 1762, 1692, 1609, 1505, 1434, 1369, 1182, 1142, 1111, 1029, 1012, 896 $\mathrm{cm}^{-1}$;

Anal. Calc'd for $\mathrm{C}_{22} \mathrm{H}_{20} \mathrm{O}_{9}$ : C, 61.68; $\mathrm{H}$, 4.71; Found: $\mathrm{C}, 61.46$; $\mathrm{H}, 4.82$;

Optical Rotation $[\alpha]_{\mathrm{D}}{ }^{24}+21.6\left(\mathrm{CHCl}_{3}, c 1.00\right)$ for $95 \%$ ee.

[16] See general methods.

[17] Recrystallization, precipitation $\left(\mathrm{Et}_{2} \mathrm{O} /\right.$ pentane $)$ and freeze-drying $\left(\mathrm{MeOH} / \mathrm{H}_{2} \mathrm{O}\right)$ were ineffective, causing partial decomposition of the product.

[18] (a) Saitoh, T.; Sakashita, S.; Nakata, H.; Shimokawa, T.; Kinjo, J.-E.; Yamahara, J.; Yamasaki, M.; Nohara, T. Chem. Pharm. Bull. 1986, 34, 2506-2511; (b) Namikoshi, M.; Nakata, H.; Yamada, H.; Nagai, M.; Saitoh, T. Chem. Pharm. Bull. 1987, 35, 2761-2773. 


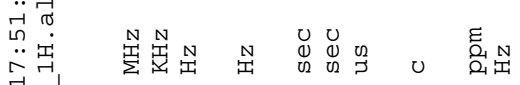

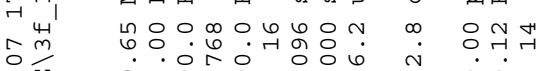

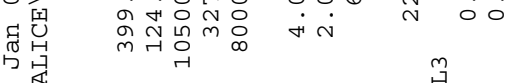

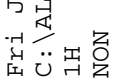

鳎

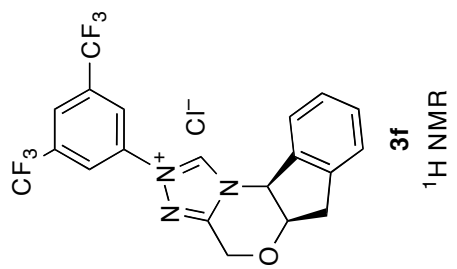

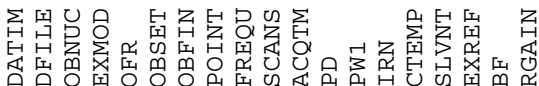


$\stackrel{\sim}{\sim} \underset{1}{\sim}$

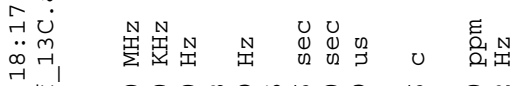

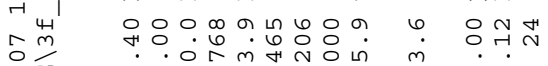

焉

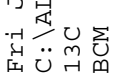

鳎

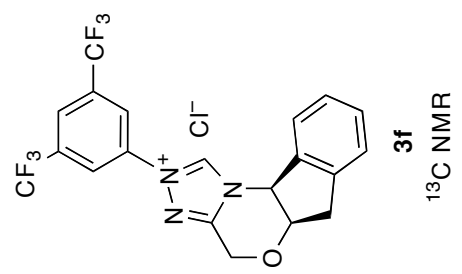

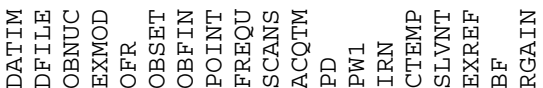


तथ

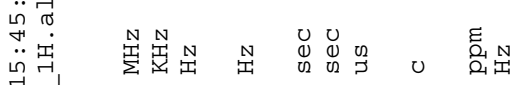

光

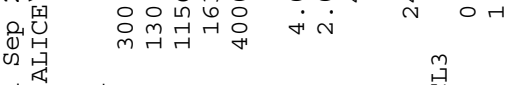

究崖出

兽

$0=\sum_{0=0}^{\infty}=0+\frac{\sum_{I}^{I}}{I}$

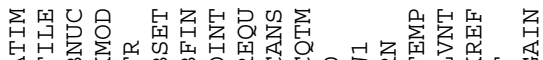

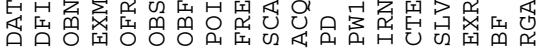

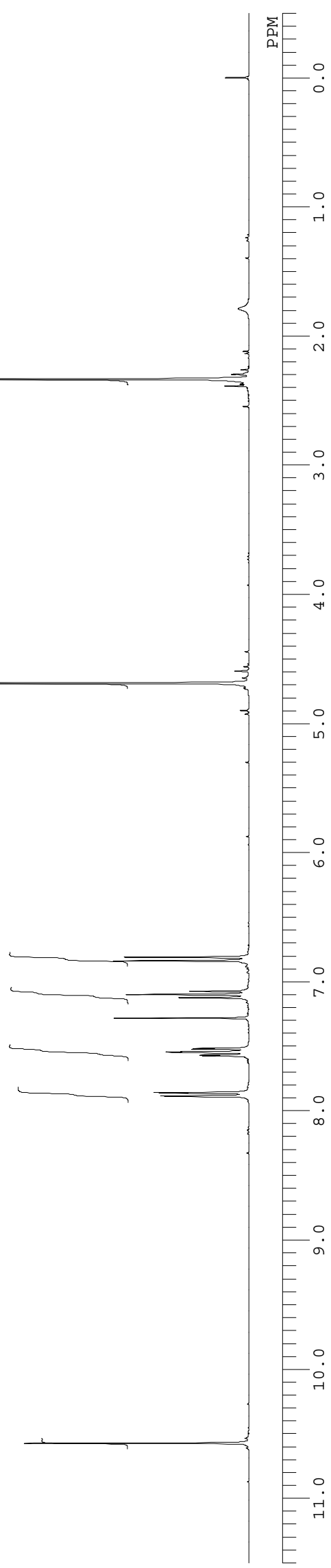




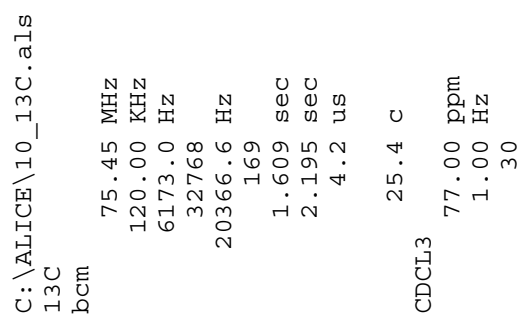

$=\sum_{0=0}^{\infty}=0$

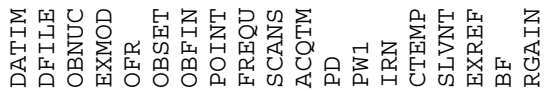

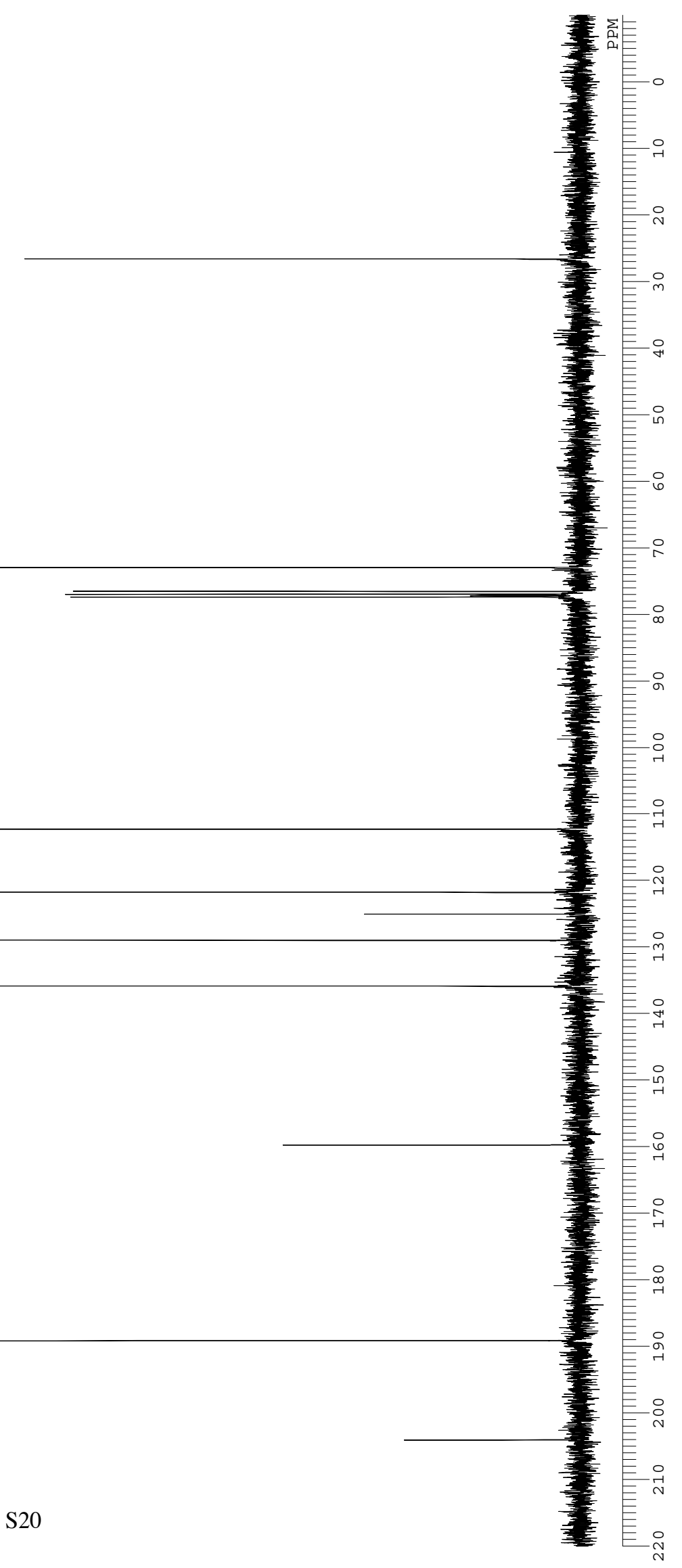


man

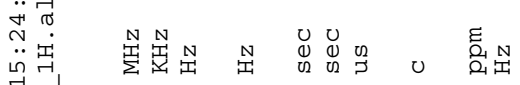

年年

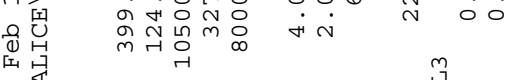

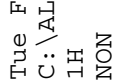

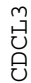

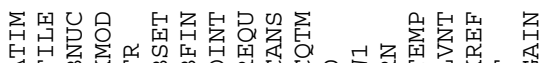

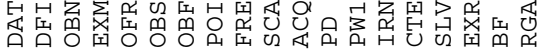

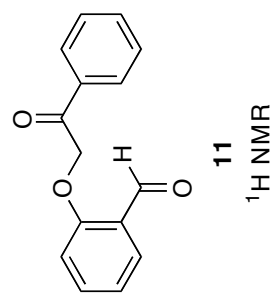


$\stackrel{\circ}{\circ}$

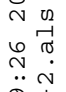

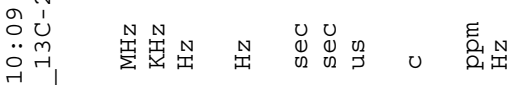



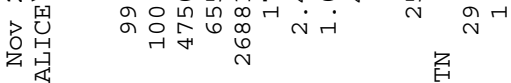

窟记

望

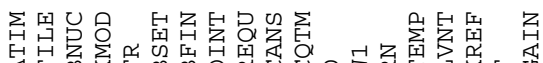

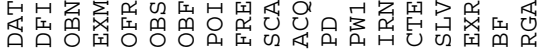

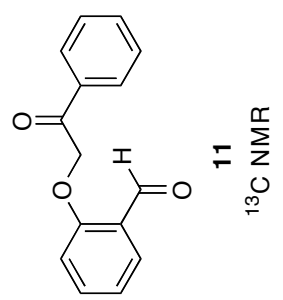

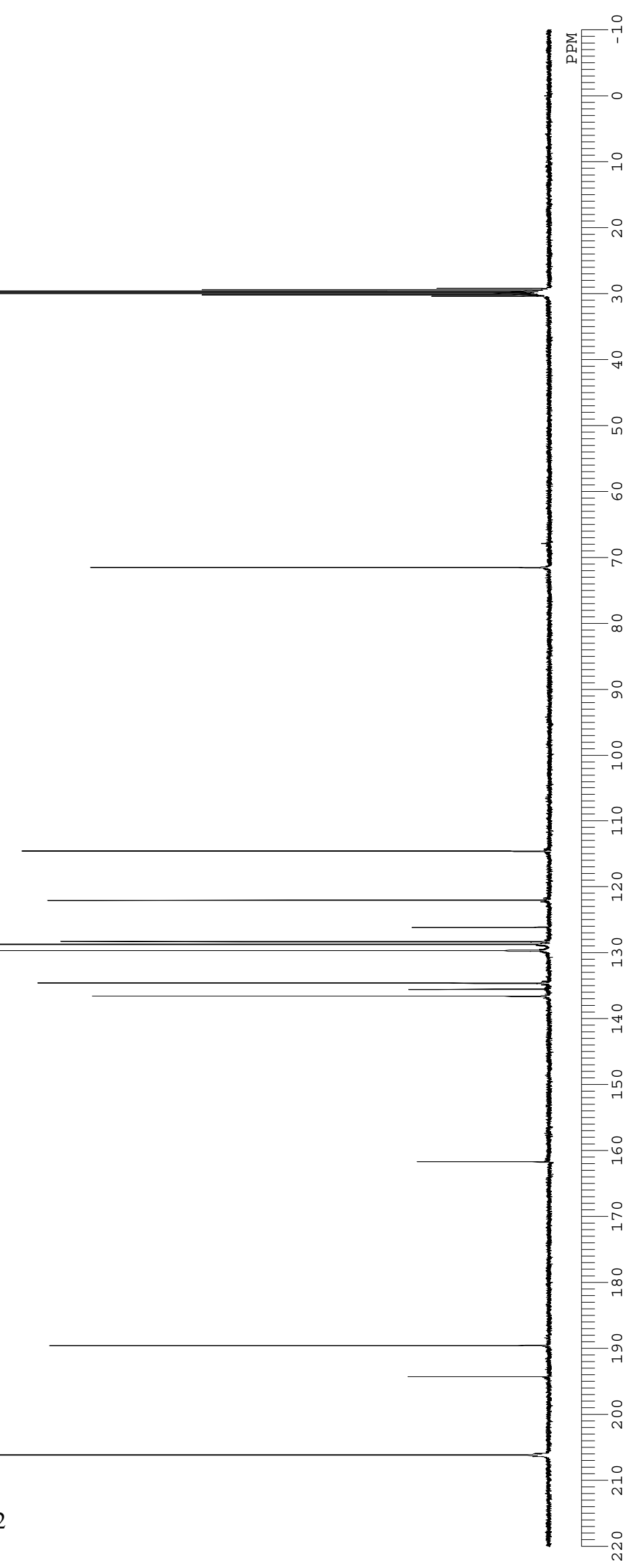




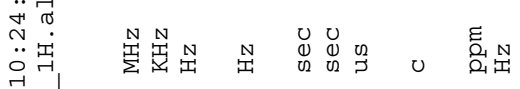

๙

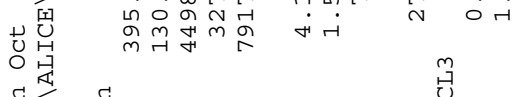

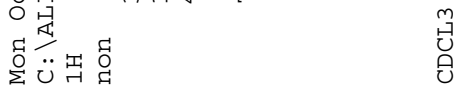

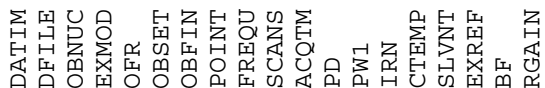

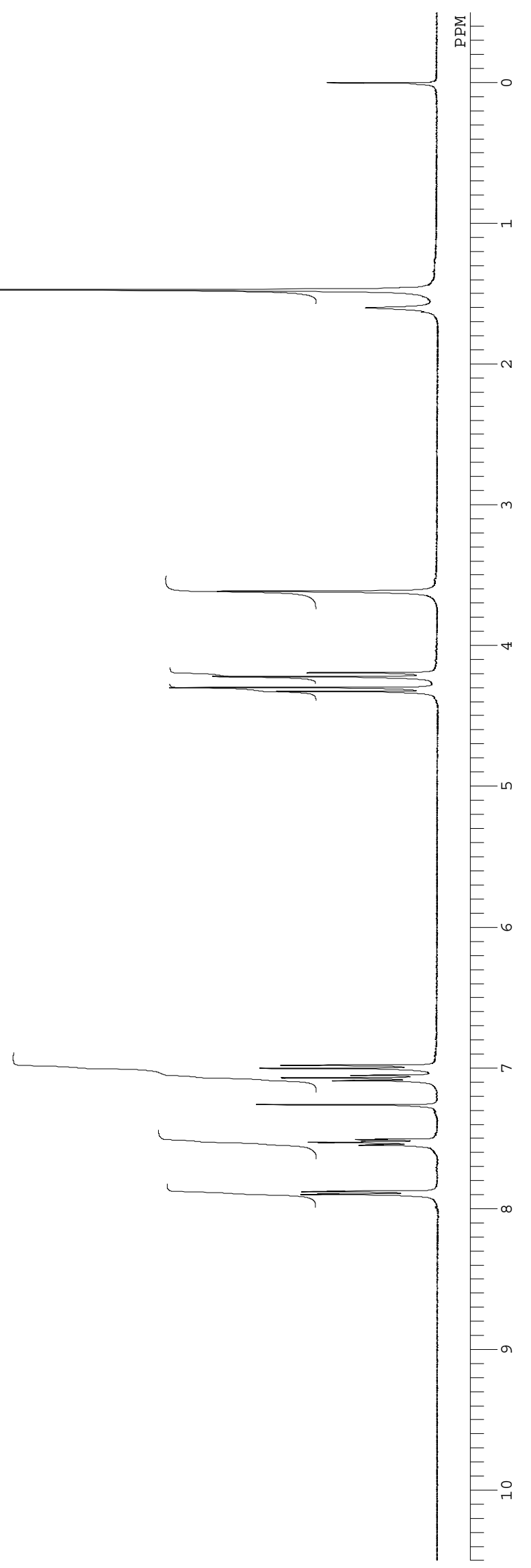




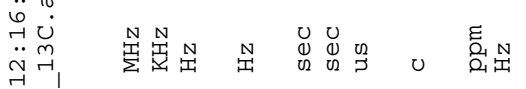

舫

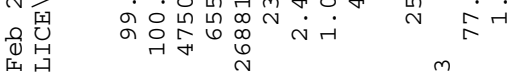

缶是U

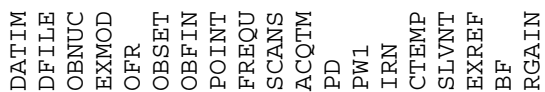

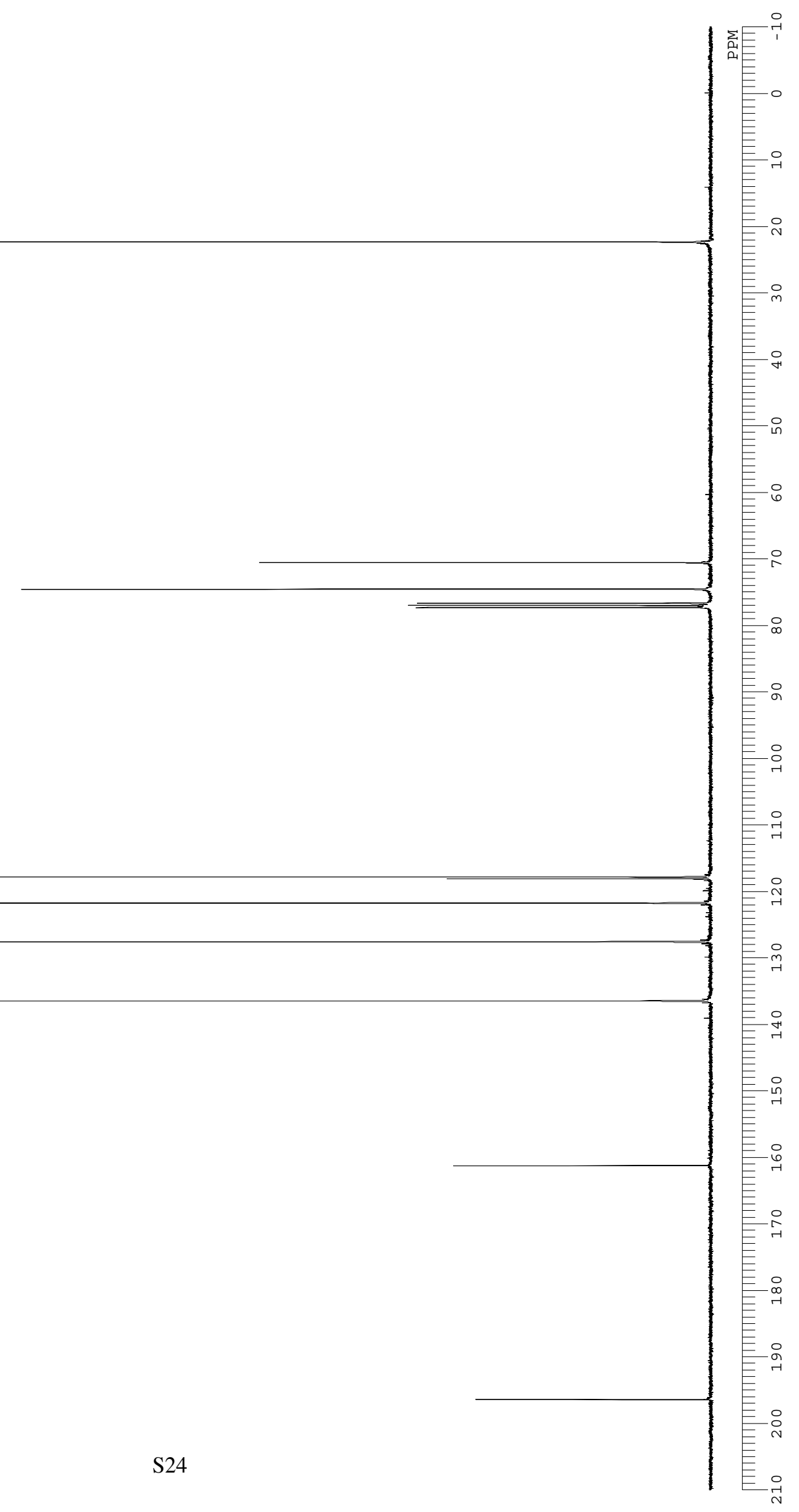


$\stackrel{\circ}{m} 0$

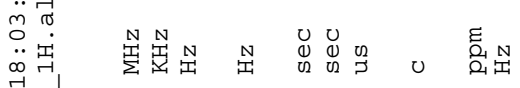

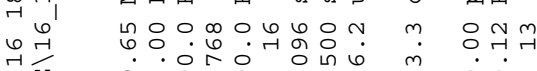

至

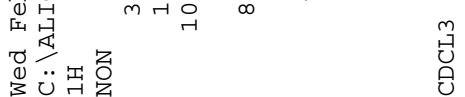

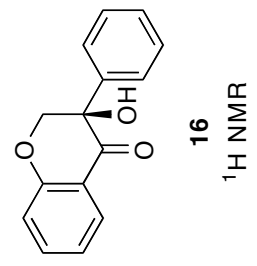

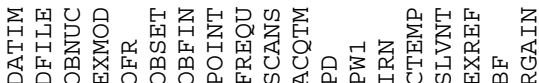

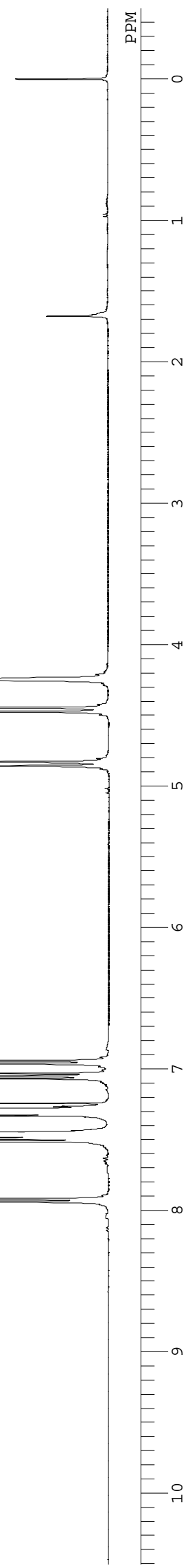




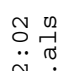

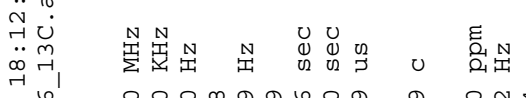

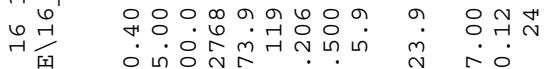

焉

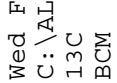

鮊

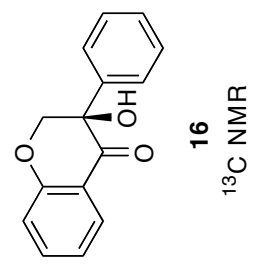

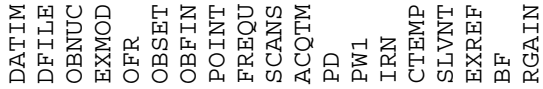

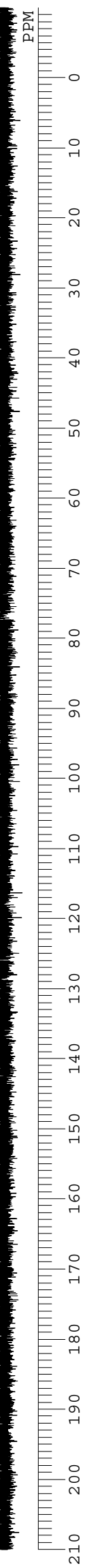




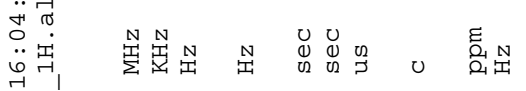

ते

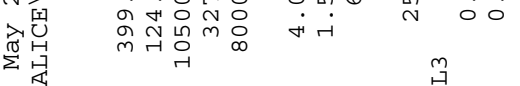

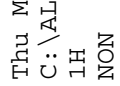

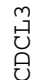

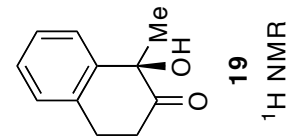

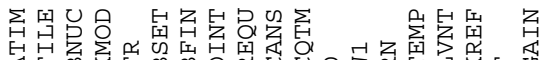

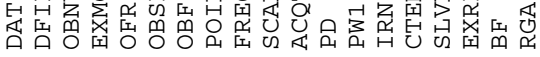

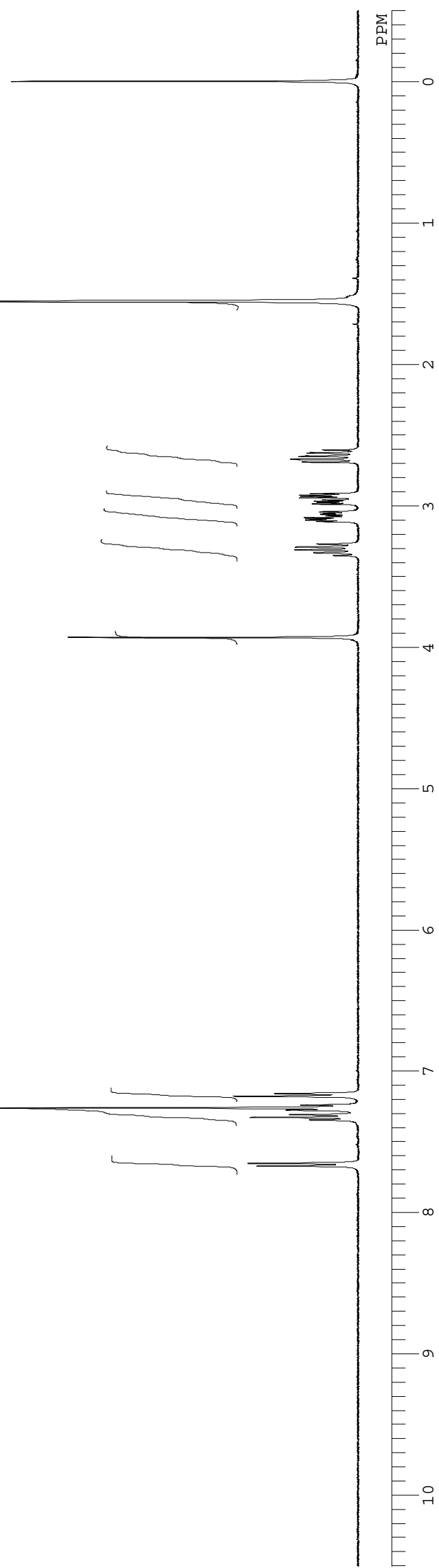




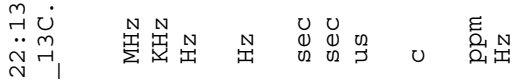

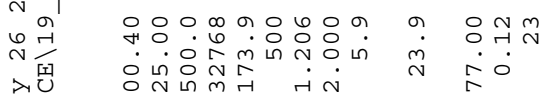

画界

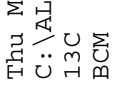

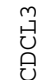

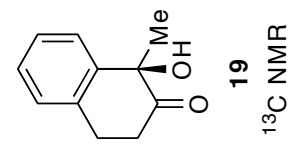

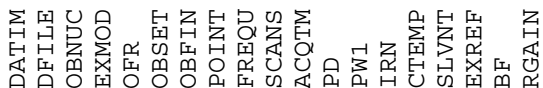

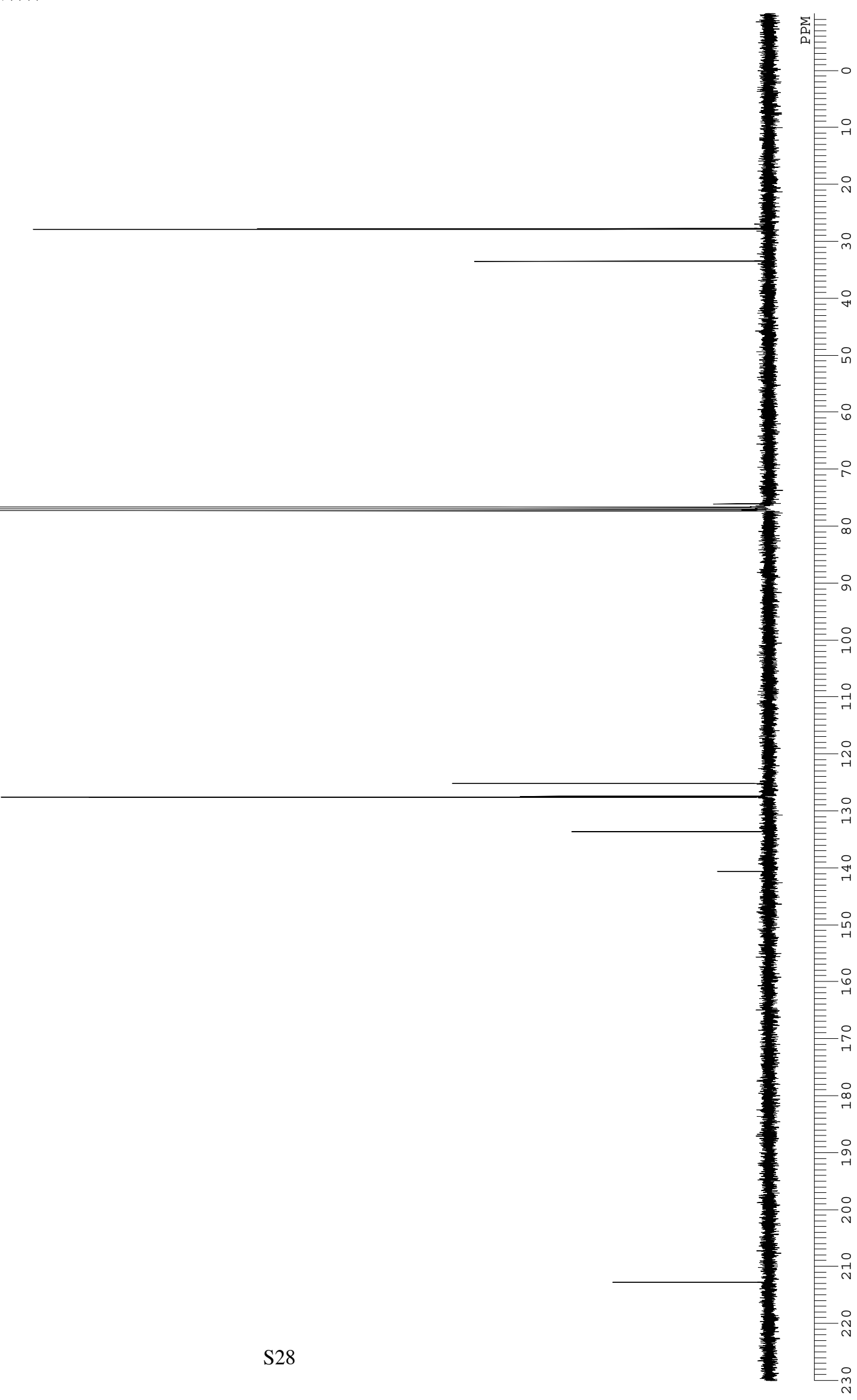




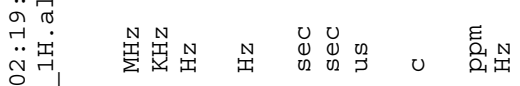

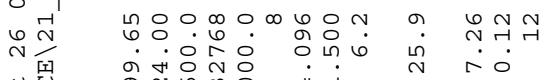

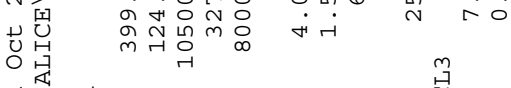

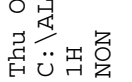

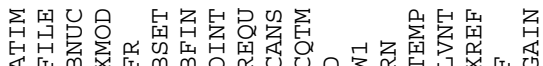

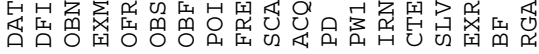




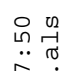

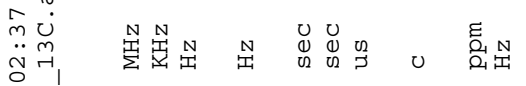

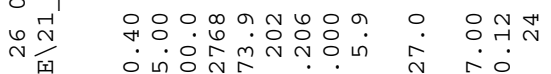

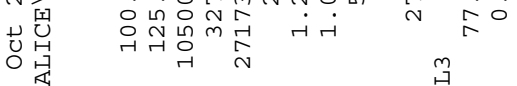

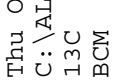

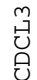

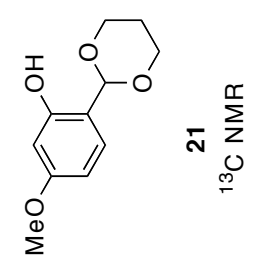

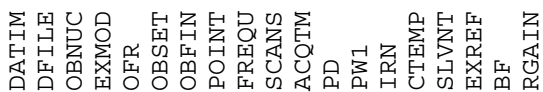

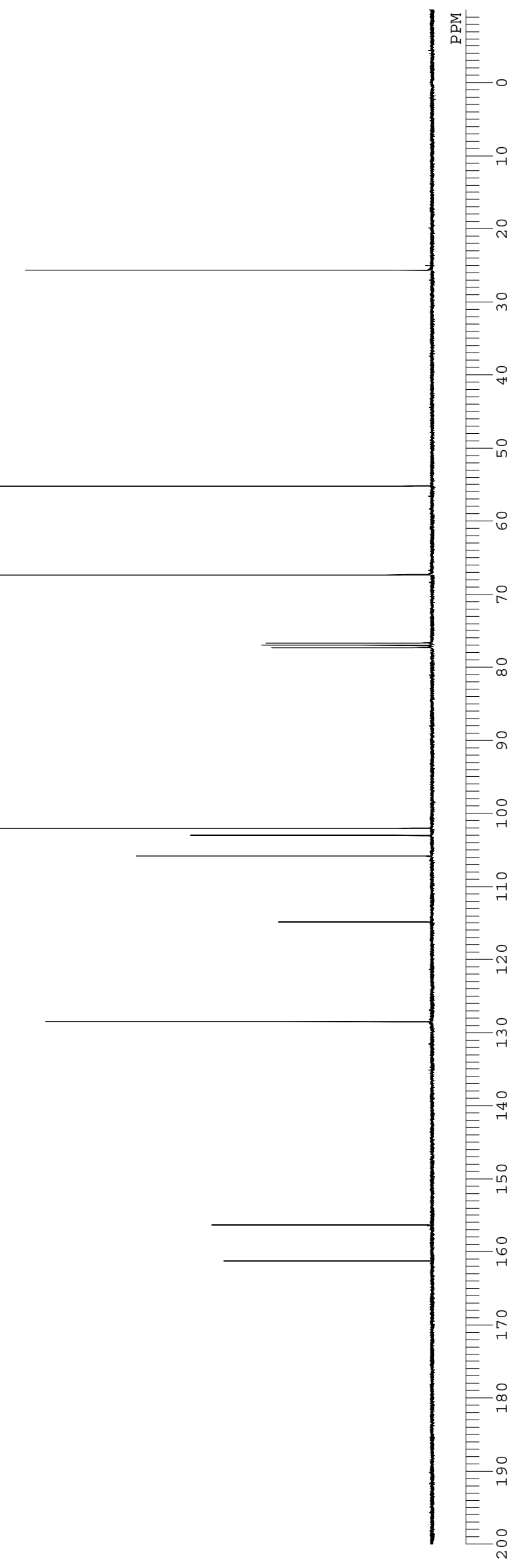




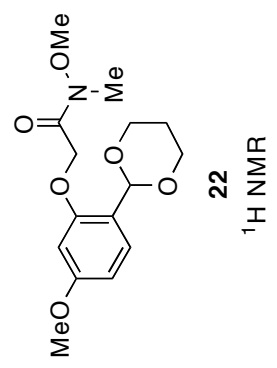

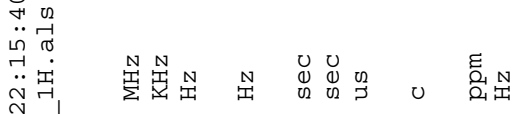

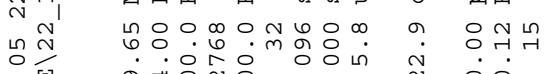

嵌

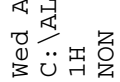

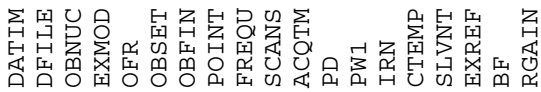

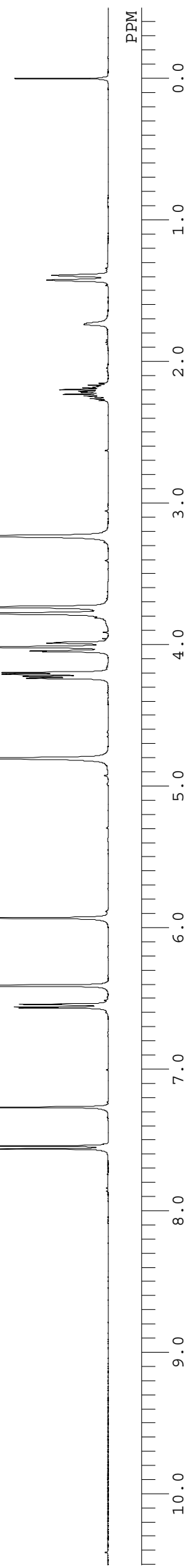




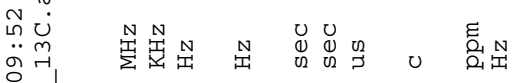

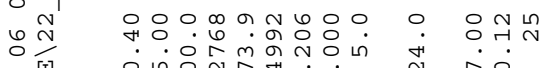

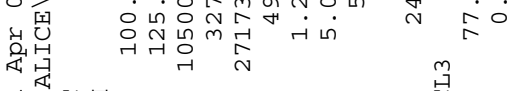

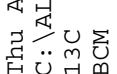

鳎

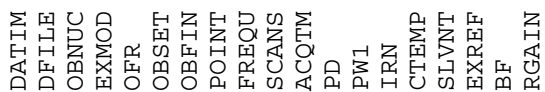

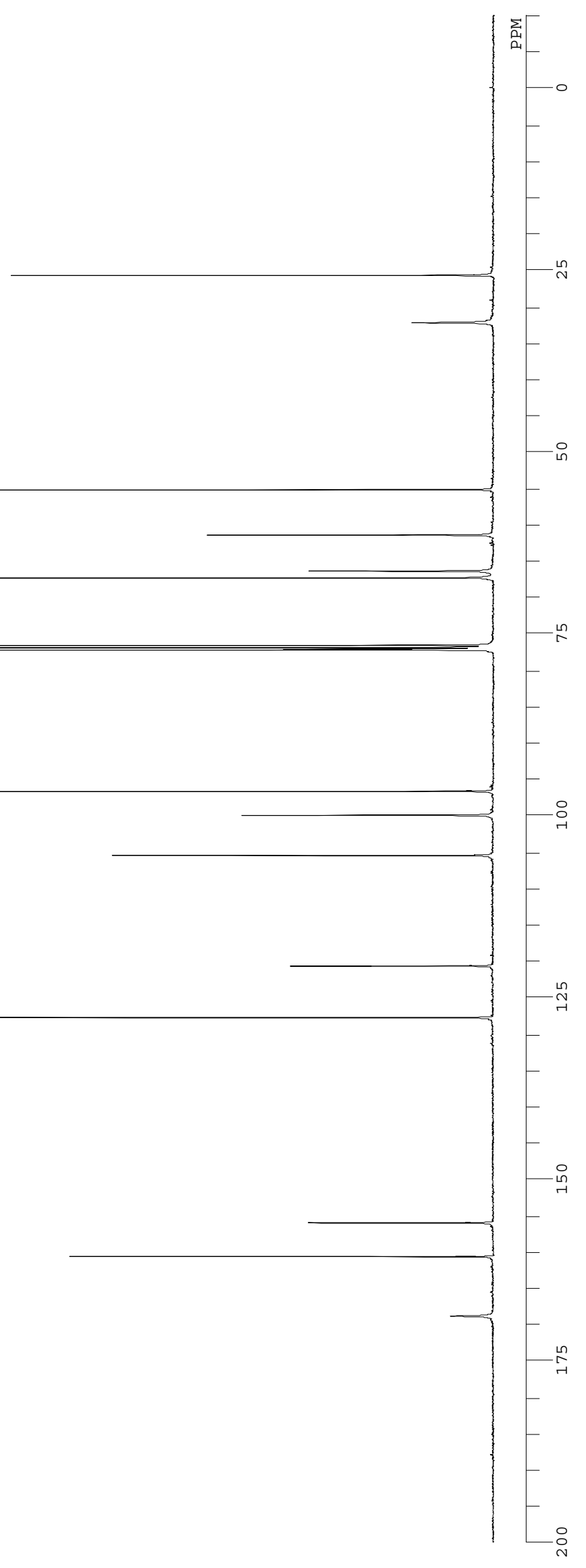




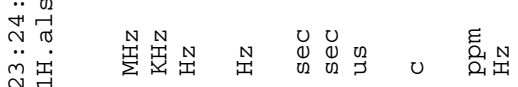

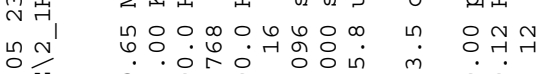

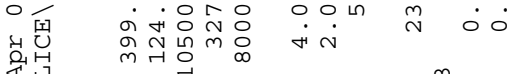

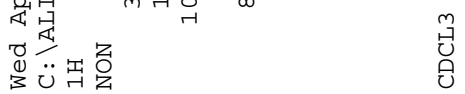

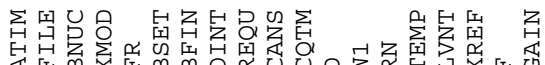

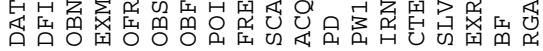

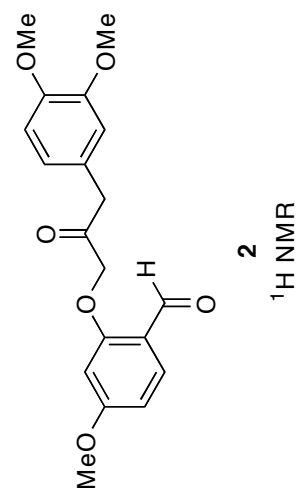




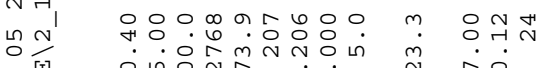

至

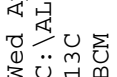

昜

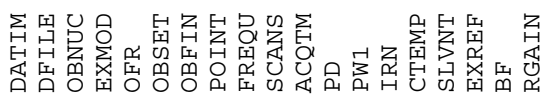
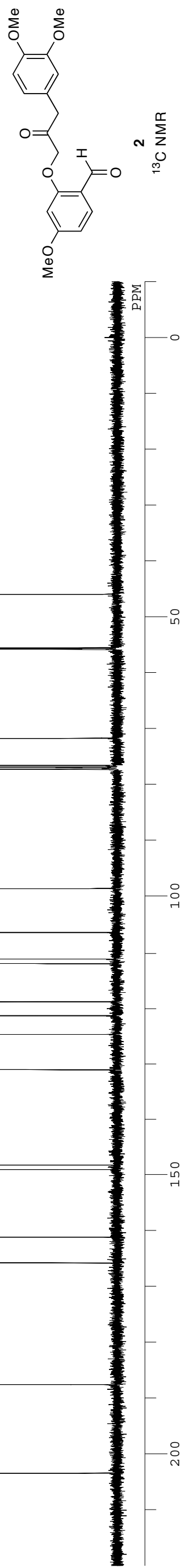


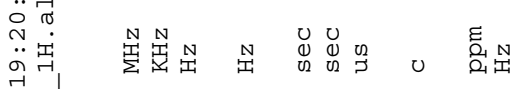

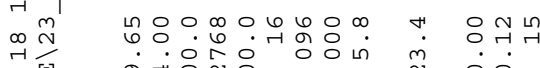

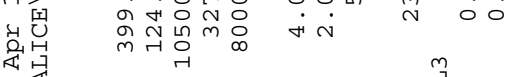

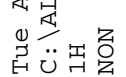

翼

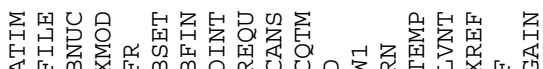

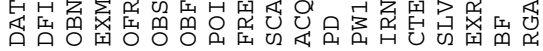
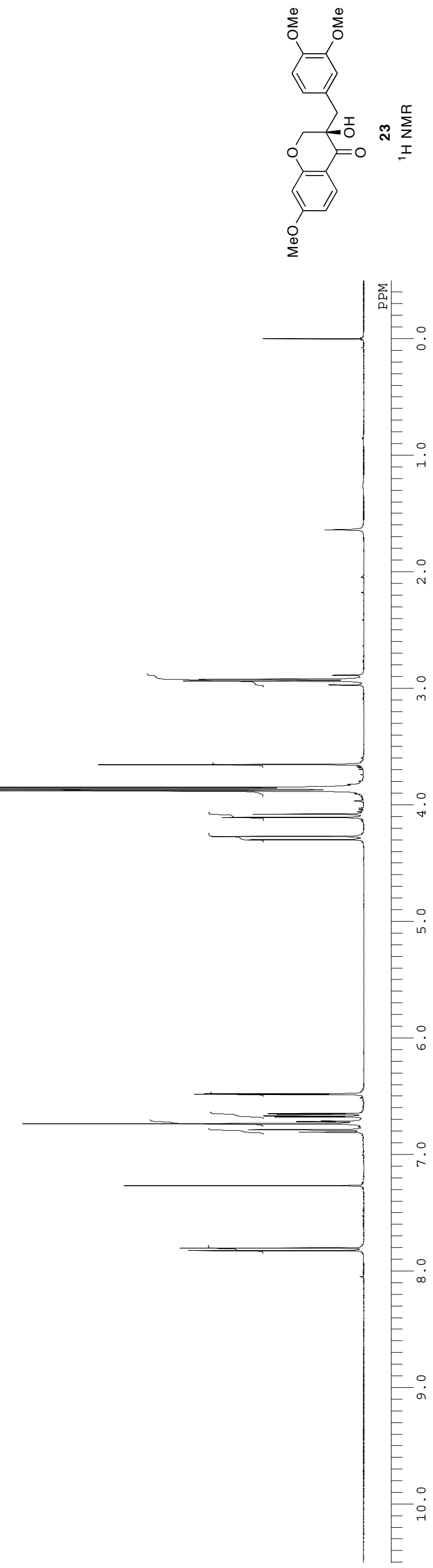


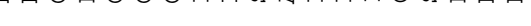

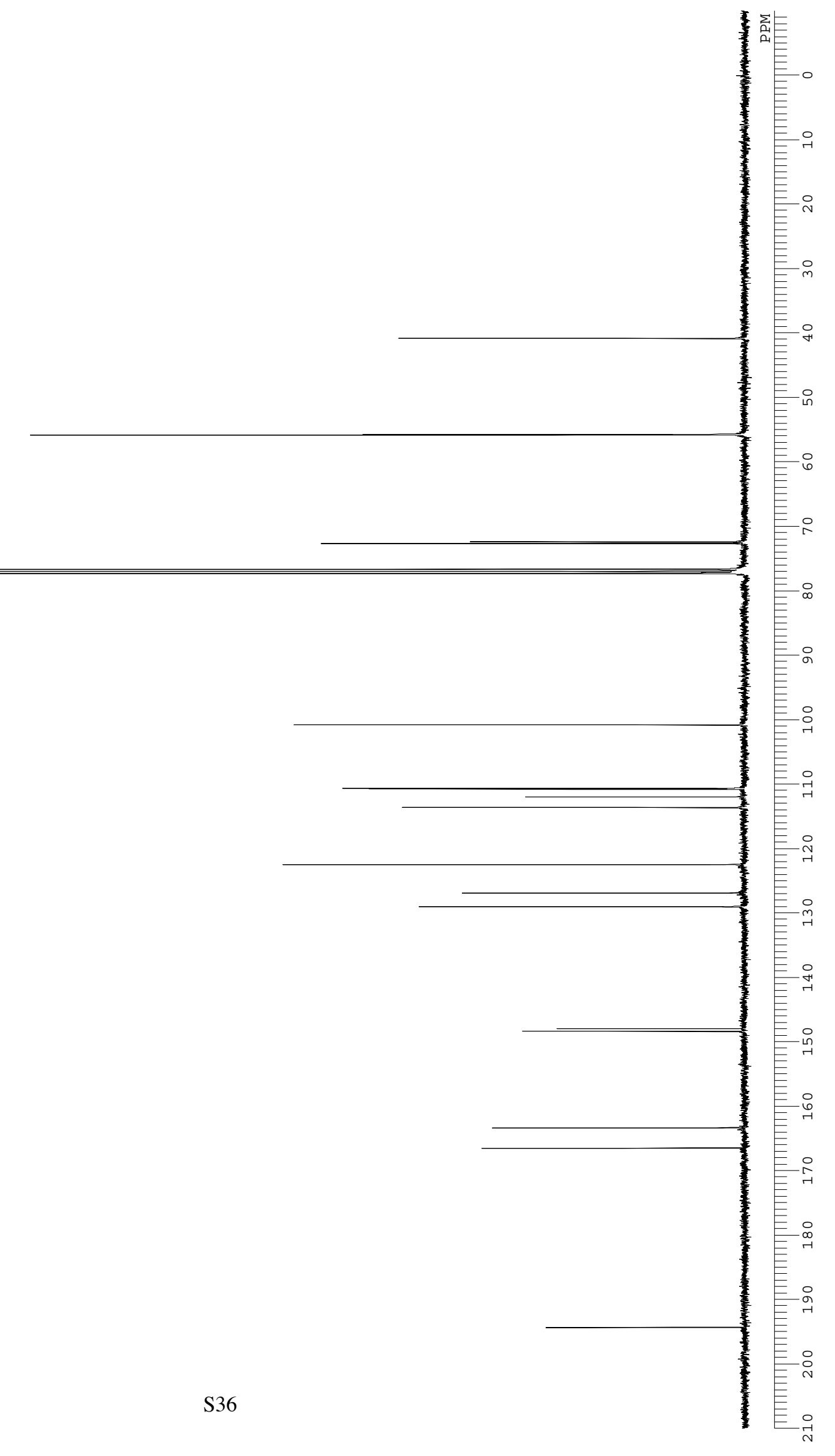




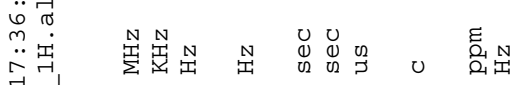

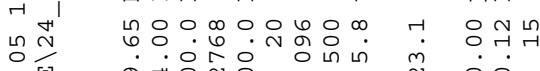

谓

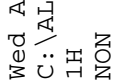

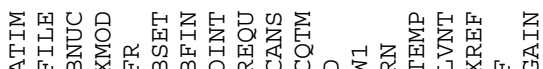

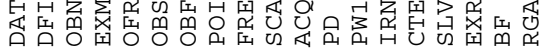
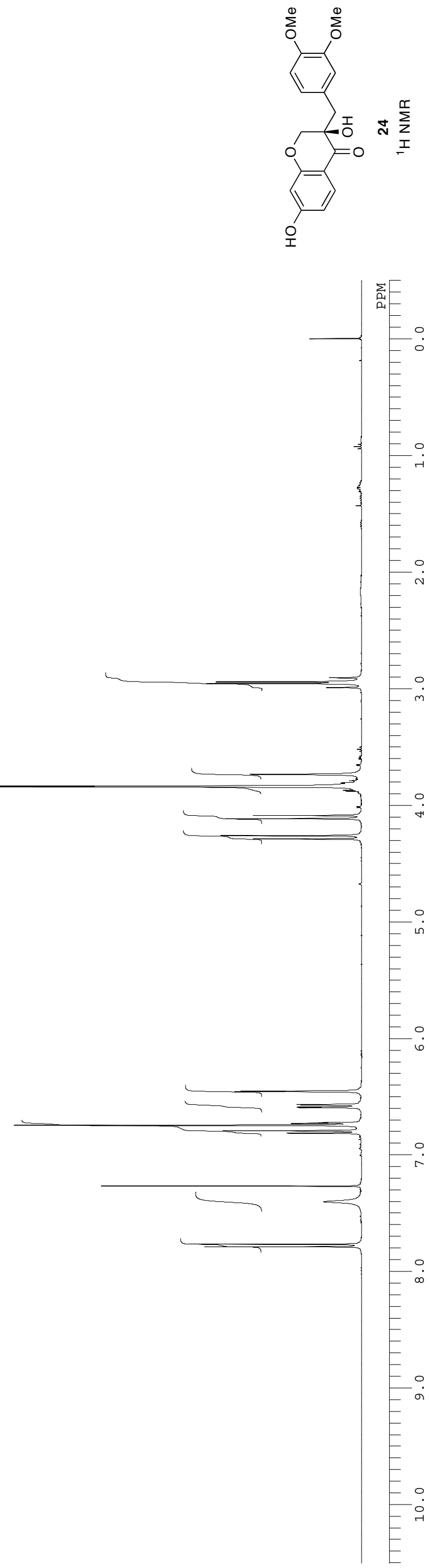


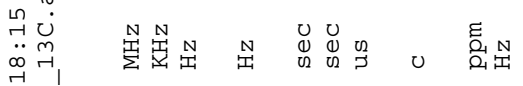

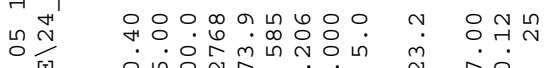

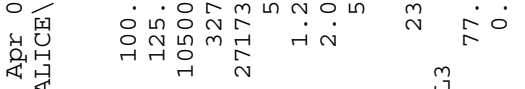

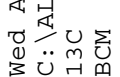

昜

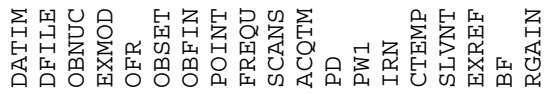
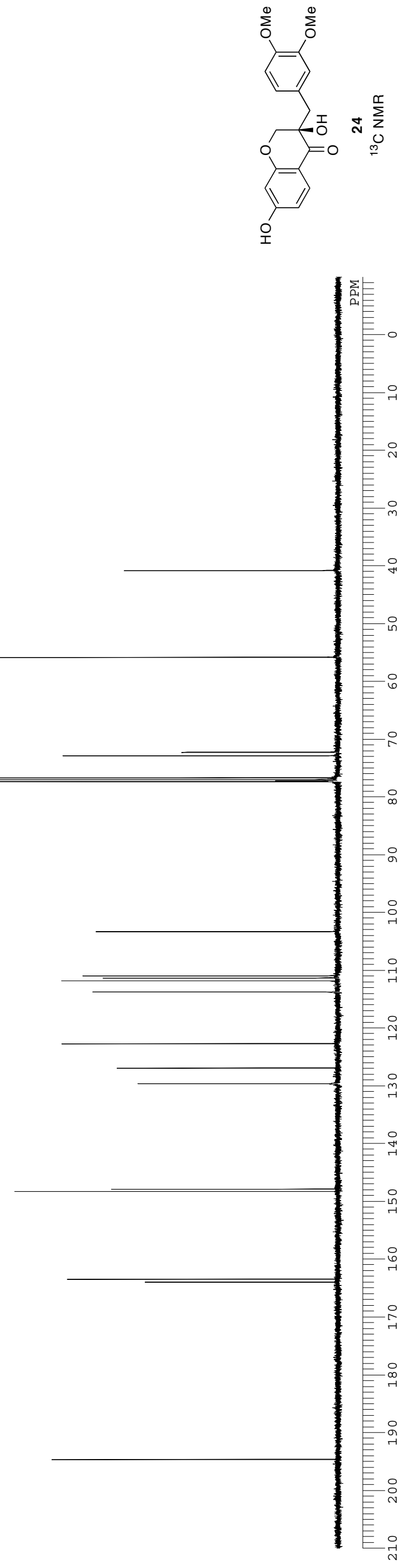


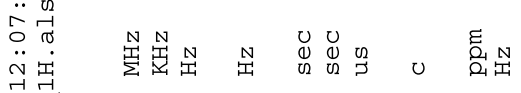

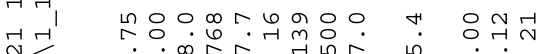

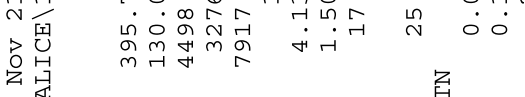

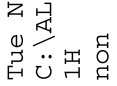

总

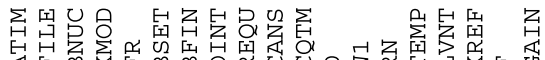

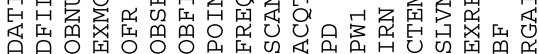
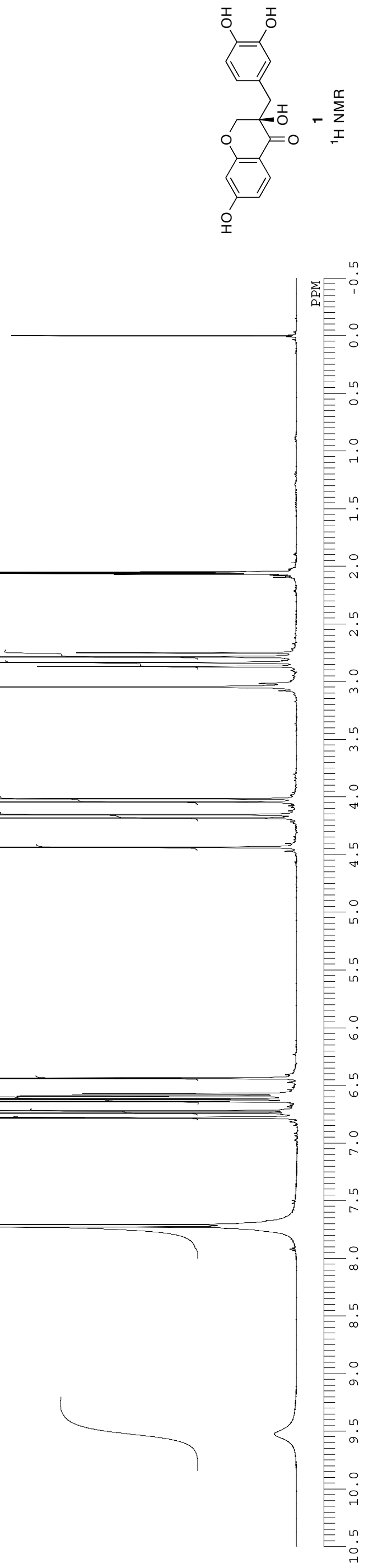


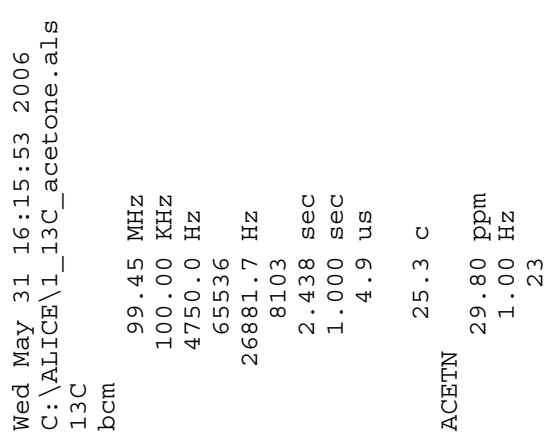

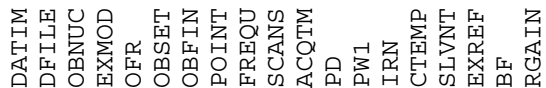
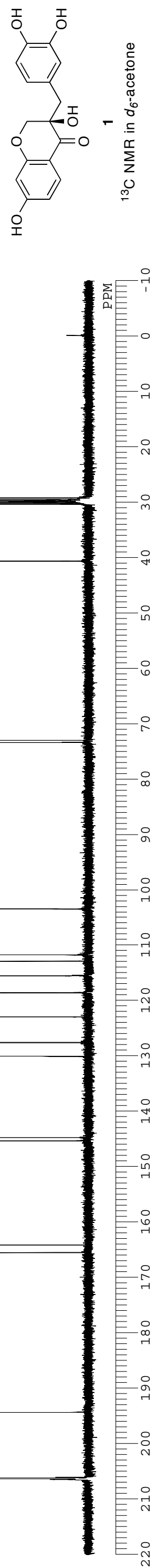


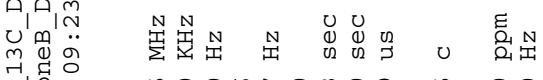

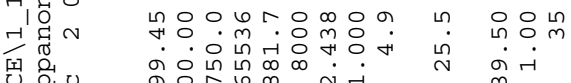

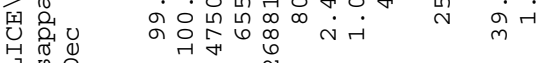

定吸

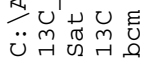

出瓷

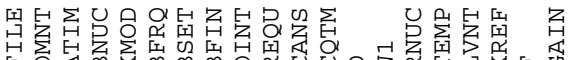

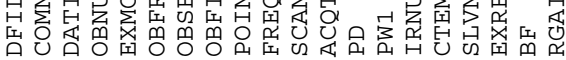
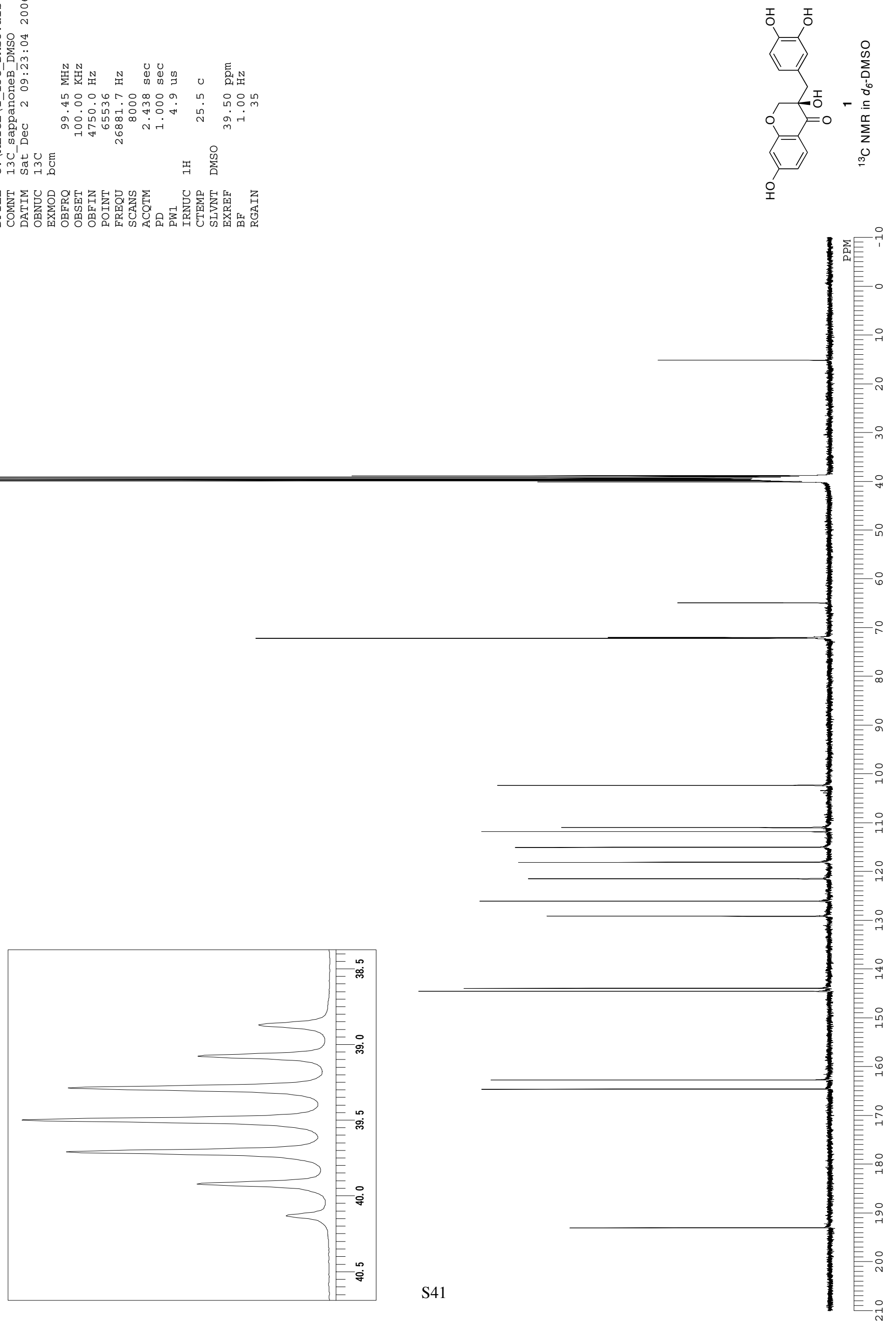


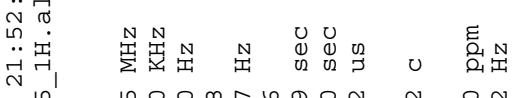

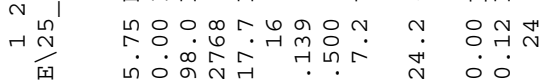

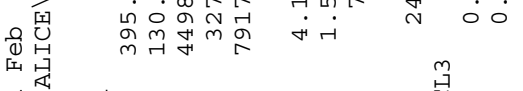

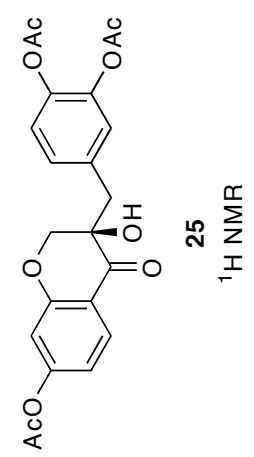

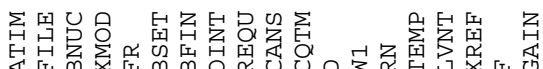

突获出

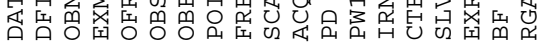

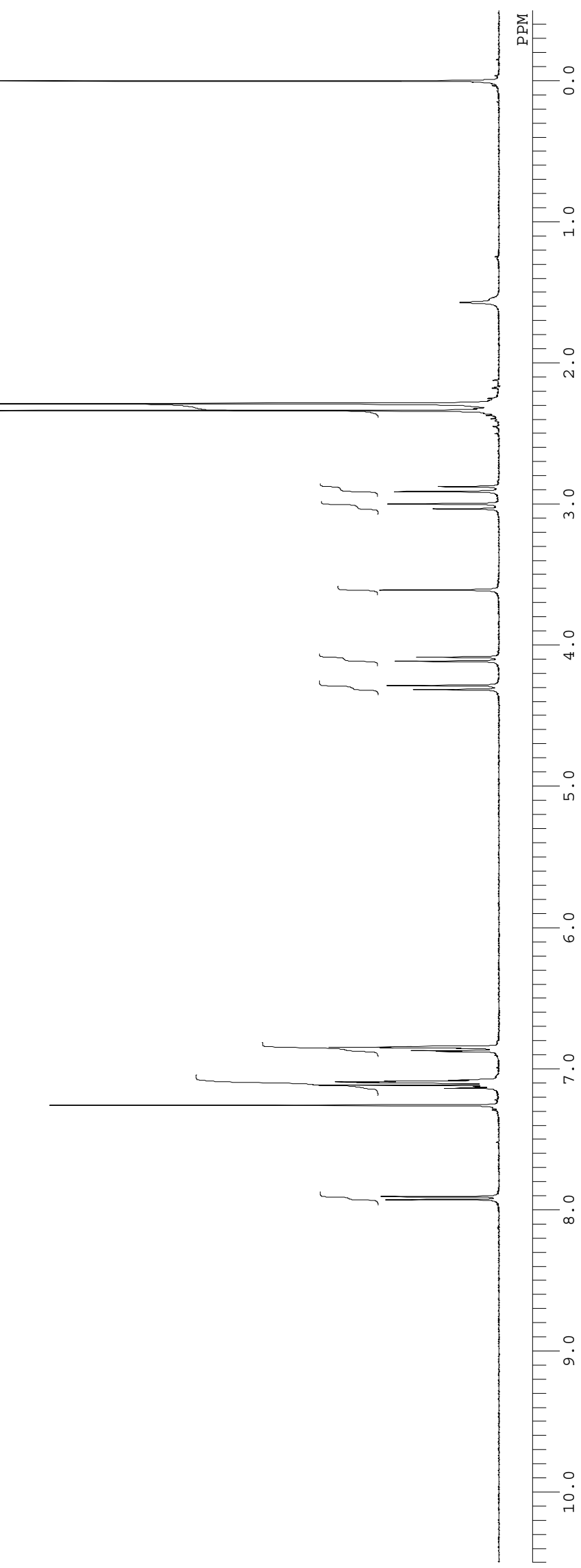




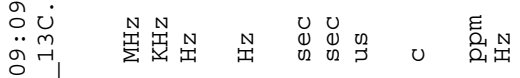

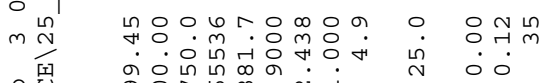

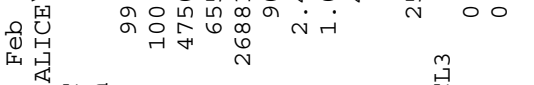

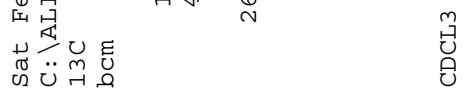

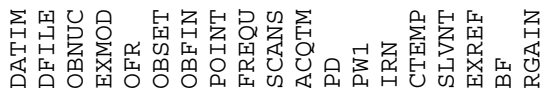
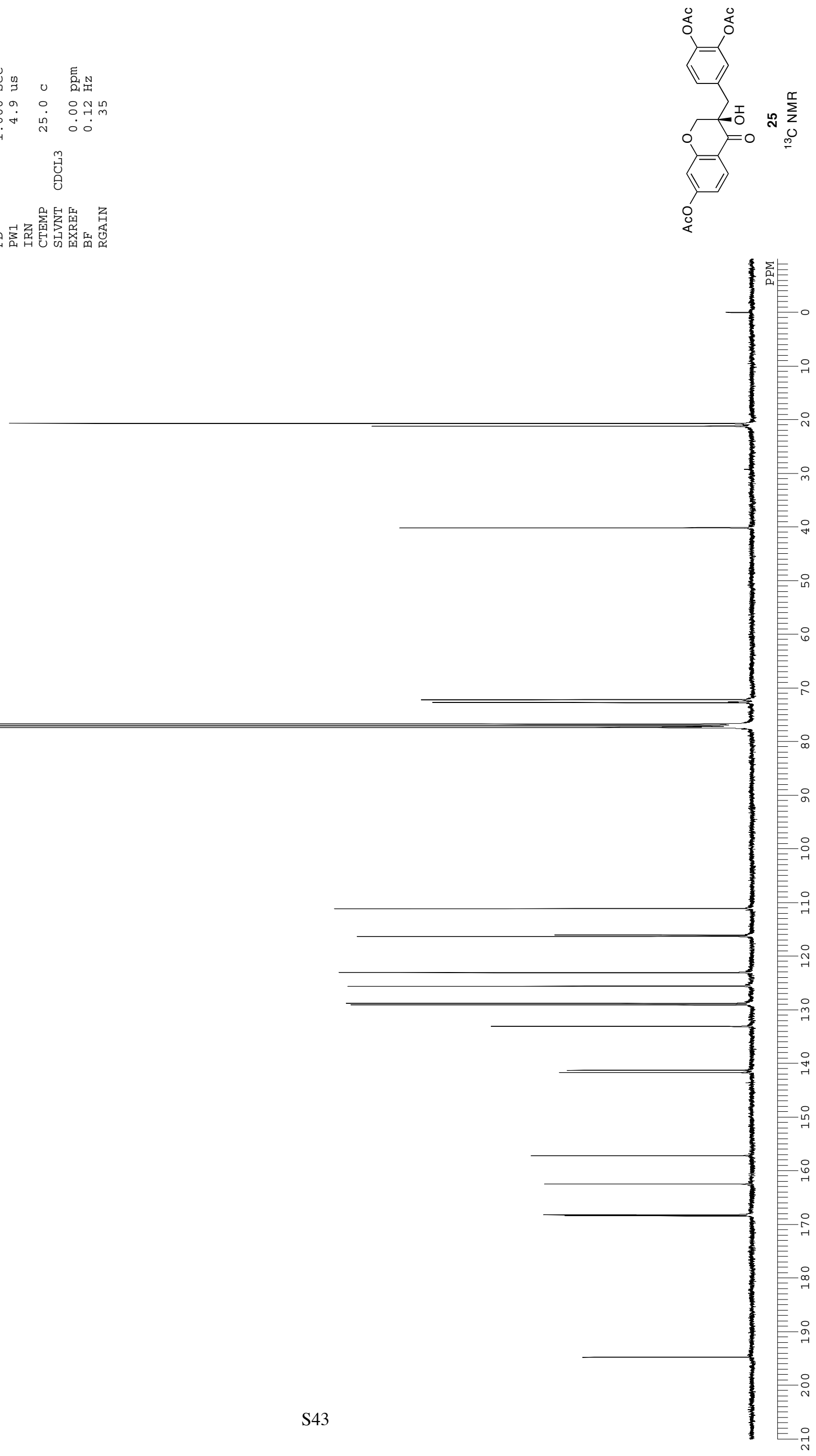